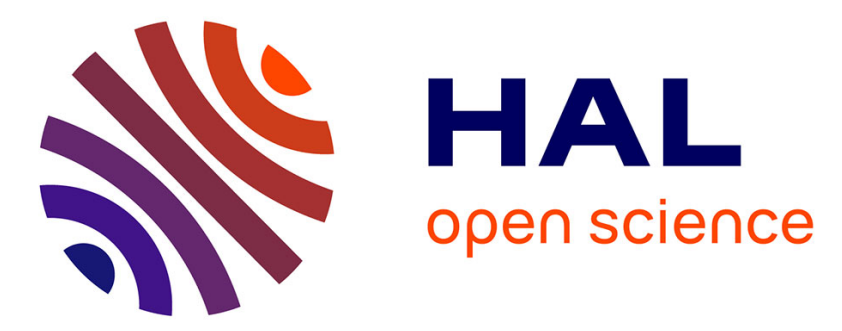

\title{
The initial-boundary value problem for general non-local scalar conservation laws in one space dimension
}

\author{
Cristiana de Filippis, Paola Goatin
}

\section{To cite this version:}

Cristiana de Filippis, Paola Goatin. The initial-boundary value problem for general non-local scalar conservation laws in one space dimension. Nonlinear Analysis: Theory, Methods and Applications, 2017, 161, pp.131-156. hal-01362504

\section{HAL Id: hal-01362504 https://hal.inria.fr/hal-01362504}

Submitted on 8 Sep 2016

HAL is a multi-disciplinary open access archive for the deposit and dissemination of scientific research documents, whether they are published or not. The documents may come from teaching and research institutions in France or abroad, or from public or private research centers.
L'archive ouverte pluridisciplinaire HAL, est destinée au dépôt et à la diffusion de documents scientifiques de niveau recherche, publiés ou non, émanant des établissements d'enseignement et de recherche français ou étrangers, des laboratoires publics ou privés. 


\title{
The initial-boundary value problem for general non-local scalar conservation laws in one space dimension
}

\author{
Cristiana De Filippis* PaOla Goatin $^{\dagger}$
}

September 8, 2016

\begin{abstract}
We prove global well-posedness results for weak entropy solutions of bounded variation (BV) of scalar conservation laws with non-local flux on bounded domains, under suitable regularity assumptions on the flux function. In particular, existence is obtained by proving the convergence of an adapted Lax-Friedrichs algorithm. Lipschitz continuos dependence from initial and boundary data is derived applying Kružhkov's doubling of variable technique.
\end{abstract}

Key words: Scalar conservation laws, Non-local flux, Initial-boundary value problem, LaxFriedrichs scheme.

\section{Introduction}

Given a bounded open interval $I=] a, b[\subset \mathbb{R}$, we consider the following initial-boundary value problem

$$
\begin{array}{ll}
\partial_{t} \rho+\partial_{x} f(t, x, \rho, \rho * \eta)=0, & (t, x) \in \mathbb{R}^{+} \times I, \\
\rho(0, x)=\rho_{0}(x), & x \in I, \\
\rho(t, a)=\rho_{a}(t), & t \in \mathbb{R}^{+}, \\
\rho(t, b)=\rho_{b}(t), & t \in \mathbb{R}^{+},
\end{array}
$$

where $f \in \mathbf{C}^{2}\left(\mathbb{R}^{+} \times \bar{I} \times \mathbb{R} \times \mathbb{R} ; \mathbb{R}\right)$ satisfies

$$
\begin{aligned}
& f(t, x, 0, R)=0 \quad \forall t, x, R, \\
& \sup _{t, x, \rho, R}\left|\partial_{\rho} f(t, x, \rho, R)\right|<L, \\
& \sup _{t, x, R}\left|\partial_{x} f(t, x, \rho, R)\right|<C|\rho|, \quad \sup _{t, x, R}\left|\partial_{R} f(t, x, \rho, R)\right|<C|\rho|, \\
& \sup _{t, x, R}\left|\partial_{x x}^{2} f(t, x, \rho, R)\right|<C|\rho|, \sup _{t, x, R}\left|\partial_{x R}^{2} f(t, x, \rho, R)\right|<C|\rho|, \sup _{t, x, R}\left|\partial_{R R}^{2} f(t, x, \rho, R)\right|<C|\rho|,
\end{aligned}
$$

for some constants $L>0$ and $C>0$, and $\eta \in\left(\mathbf{C}^{\mathbf{1}} \cap \mathbf{W}^{1, \infty}\right)(\mathbb{R} ; \mathbb{R}$ ) is a convolution kernel (not necessarily with compact support) such that

$$
\int_{\mathbb{R}} \eta(x) d x=1
$$

Equations of type (1.1a) arise in several applications, and have made the object of a large literature in recent years. Space-integral terms appear for example in models for granular flows

\footnotetext{
${ }^{*}$ University of Milano-Bicocca, Italy, \& Inria Sophia Antipolis - Méditerranée, France, E-mail: c.defilippis@campus.unimib.it

${ }^{\dagger}$ Inria Sophia Antipolis - Méditerranée, France, E-mail: paola.goatin@inria.fr
} 
[3], sedimentation [7], supply chains [19], conveyor belts [18], weakly coupled oscillators [2], structured populations dynamics [24], or more general problems like gradient constrained equations [4]. Equations with non-local flux have been recently introduced also in traffic flow modeling to account for the reaction of drivers or pedestrians to the surrounding density of other individuals, see $[8,10,11,26]$.

General analytical results on non-local conservation laws, proving existence and eventually uniqueness of solutions of the Cauchy problem for (1.1a), can be found in [5] for scalar equations in one space dimension, in [12] for scalar equations in several space dimensions and in $[1,13,14]$ for multidimensional systems of conservation laws. Besides, specific finite volume numerical methods have been developed recently in $[1,17,21]$. To our knowledge, initial-boundary value problems of the form (1.1) have not been rigorously studied yet, the difficulties lying in the presence of the nonlocal term, which may exceed the boundaries of the space domain. Nonetheless, real applications (confined environments, networks, etc.) and numerical computations require a precise account for boundary conditions.

The scope of the present article is to propose an approach for a rigorous treatment of boundary conditions, in the case of one space-dimensional problems. The strategies we employ are inspired by classical results on scalar conservation laws with boundary conditions. In particular, we refer to $[6,9,27]$. Our results are based on the extension of the solution outside the domain, set to be constantly equal to the corresponding boundary condition values. It is far from obvious to generalize this technique to problem in several space-dimensions.

As in the classical case, we assume that boundary conditions can not generally be satisfied in strong sense. Therefore, we introduce the following notion of weak entropy solution for (1.1), which extends to problems with boundaries the definition of solution given in [5] for the corresponding Cauchy problem. This formulation, based on semi Kružhkov entropies [23, 27], has the advantage of not using explicitly the traces of the solution at the boundaries of the domain, which turns particularly useful in the existence proof, provided in Section 2.

Definition 1 Let $\rho_{0} \in \mathbf{L}^{\infty}(I ; \mathbb{R})$ and $\rho_{a}, \rho_{b} \in \mathbf{L}^{\infty}\left(\mathbb{R}^{+} ; \mathbb{R}\right)$. A map $\rho \in \mathbf{L}^{\infty}\left(\mathbb{R}^{+} \times I ; \mathbb{R}\right)$ is a weak entropy solution to (1.1) if for every test function $\varphi \in \mathbf{C}_{\mathbf{c}}^{\mathbf{1}}\left(\mathbb{R}^{2} ; \mathbb{R}^{+}\right)$and for every $\kappa \in \mathbb{R}$

$$
\begin{gathered}
\int_{0}^{+\infty} \int_{a}^{b}\left((\rho-\kappa)^{ \pm} \partial_{t} \varphi+\operatorname{sgn}(\rho-\kappa)^{ \pm}(f(t, x, \rho, R(t, x))-f(t, x, \kappa, R(t, x))) \partial_{x} \varphi\right. \\
\left.\quad-\operatorname{sgn}(\rho-\kappa)^{ \pm} \frac{d}{d x} f(t, x, \kappa, R(t, x)) \varphi\right) d x d t+\int_{a}^{b}\left(\rho_{0}-\kappa\right)^{ \pm} \varphi(0, x) d x \\
+\operatorname{Lip}(f) \int_{0}^{+\infty}\left(\rho_{a}(t)-\kappa\right)^{ \pm} \varphi(t, a) d t+\operatorname{Lip}(f) \int_{0}^{+\infty}\left(\rho_{b}(t)-\kappa\right)^{ \pm} \varphi(t, b) d t \geq 0
\end{gathered}
$$

where

$$
\begin{aligned}
R(t, x):=(\rho(t, \cdot) * \eta)(x) & =\int_{\mathbb{R}} \rho(t, y) \eta(x-y) d y \\
& =\int_{a}^{b} \rho(t, y) \eta(x-y) d y+\rho_{a}(t) \int_{-\infty}^{a} \eta(x-y) d y+\rho_{b}(t) \int_{b}^{+\infty} \eta(x-y) d y \\
& =\int_{a}^{b} \rho(t, y) \eta(x-y) d y+\rho_{a}(t) \int_{x-a}^{+\infty} \eta(y) d y+\rho_{b}(t) \int_{-\infty}^{x-b} \eta(y) d y . \quad(1.5)
\end{aligned}
$$

Above, we have noted $\operatorname{sgn}^{+}(s):=\max \{s /|s|, 0\}, \operatorname{sgn}^{-}(s):=-\operatorname{sgn}^{+}(-s), s^{+}:=s \operatorname{sgn}^{+}(s)$ and $s^{-}:=(-s)^{+}$for $s \in \mathbb{R}$. In the paper, we will also denote $\mathcal{I}(r, s):=[\min \{r, s\}, \max \{r, s\}]$ for any $r, s \in \mathbb{R}$. 
The Definition 1 is equivalent to the one provided in [6] (for a proof of equivalence we refer the reader to [22, Theorem 7.31]). This second definition will be used in Section 3 to prove Lipschitz continuous dependence of solution with respect to initial and boundary data.

Definition 2 Let $\rho_{0} \in \mathbf{L}^{\infty}(I ; \mathbb{R})$ and $\rho_{a}, \rho_{b} \in \mathbf{L}^{\infty}\left(\mathbb{R}^{+} ; \mathbb{R}\right)$. A map $\rho \in B V\left(\mathbb{R}^{+} \times I ; \mathbb{R}\right)$ is a weak entropy solution to (1.1) if for every test function $\varphi \in \mathbf{C}_{\mathbf{c}}^{\mathbf{1}}\left(\mathbb{R}^{2} ; \mathbb{R}^{+}\right)$and for every $\kappa \in \mathbb{R}$

$$
\begin{gathered}
\int_{0}^{+\infty} \int_{a}^{b}\left(|\rho-\kappa| \partial_{t} \varphi+\operatorname{sgn}(\rho-\kappa)[f(t, x, \rho, R(t, x))-f(t, x, \kappa, R(t, x))] \partial_{x} \varphi\right. \\
\left.\quad-\operatorname{sgn}(\rho-\kappa) \frac{d}{d x} f(t, x, \kappa, R(t, x)) \varphi\right) d x d t+\int_{a}^{b}\left|\rho_{0}-\kappa\right| \varphi(0, x) d x \\
+\int_{0}^{+\infty} \operatorname{sgn}\left(\rho_{a}-\kappa\right)[f(t, a, \rho(t, a+), R(t, a))-f(t, a, \kappa, R(t, a))] \varphi(t, a) d t \\
\quad+\int_{0}^{\infty} \operatorname{sgn}\left(\rho_{b}-\kappa\right)[f(t, b, \kappa, R(t, b))-f(t, b, \rho(t, b-), R(t, b))] \varphi(t, b) d t \geq 0 .
\end{gathered}
$$

We remark that to ensure that the traces of $\rho$ at $x=a, b$, are well defined, we need to assume that the solutions have bounded variation, see [6, Lemma 1]. Moreover, following $[6,15]$, we recall that the entropy condition (1.6) implies that the traces of the solution at the boundary satisfy

- On the left boundary $x=a$ : for all $\kappa \in \mathbb{R}$

$$
\left(\operatorname{sgn}(\rho(t, a+)-\kappa)-\operatorname{sgn}\left(\rho_{a}(t)-\kappa\right)\right)(f(t, a, \rho(t, a+), R(t, a))-f(t, a, \kappa, R(t, a))) \leq 0,
$$

- On the right boundary $x=b$ : for all $\kappa \in \mathbb{R}$

$$
\left(\operatorname{sgn}(\rho(t, b-)-\kappa)-\operatorname{sgn}\left(\rho_{b}(t)-\kappa\right)\right)(f(t, b, \rho(t, b-), R(t, b))-f(t, b, \kappa, R(t, b))) \geq 0 .
$$

Our main result states the global well-posedness of (1.1).

Theorem 1 Let hypotheses (1.2) hold. If $\rho_{0} \in\left(\mathbf{L}^{\infty} \cap B V\right)\left(I ; \mathbb{R}^{+}\right)$and $\rho_{a}, \rho_{b} \in\left(\mathbf{L}^{\infty} \cap B V\right)\left(\mathbb{R}^{+} ; \mathbb{R}^{+}\right)$, then for all $T>0$ problem (1.1) has a unique weak entropy solution $\rho \in B V\left([0, T] \times I ; \mathbb{R}^{+}\right)$in the sense of Definitions 1, 2. Moreover, the following estimates hold:

$$
\begin{aligned}
& \|\rho(T, \cdot)\|_{\mathbf{L}^{1}(I)} \leq\left\|\rho_{0}\right\|_{\mathbf{L}^{\mathbf{1}}(I)}+\alpha\left(\left\|\rho_{a}\right\|_{\mathbf{L}^{\mathbf{1}}([0, T])}+\left\|\rho_{b}\right\|_{\mathbf{L}^{\mathbf{1}}([0, T])}\right), \\
& \|\rho(T, \cdot)\|_{\mathbf{L}^{\infty}(I)} \leq e^{\mathcal{L} T}\left\|\rho_{0}\right\|_{\mathbf{L}^{\infty}(I)}, \\
& T V(\rho(T, \cdot) ; I) \leq e^{\mathcal{K}_{1} T} T V\left(\rho_{0} ; I\right)+\frac{\mathcal{K}_{2}}{\mathcal{K}_{1}}\left(e^{\mathcal{K}_{1} T}-1\right)+T V\left(\rho_{a} ;[0, T]\right)+T V\left(\rho_{b} ;[0, T]\right), \\
& \|\rho(T, \cdot)-\rho(T-\tau, \cdot)\|_{\mathbf{L}^{1}(I)} \leq \mathcal{C}_{t}(T) \tau, \quad \tau>0,
\end{aligned}
$$

with $\mathcal{L}$ as in (2.12), $\mathcal{K}_{1,2}$ as in (2.18) and (2.24), and $\mathcal{C}_{t}$ as in (2.28).

Finally, let $\rho, \sigma \in \mathbf{C}^{\mathbf{0}}\left(\mathbb{R}^{+} ; \mathbf{L}^{\mathbf{1}}\left(I ; \mathbb{R}^{+}\right)\right) \cap B V \infty\left([0, T] \times I ; \mathbb{R}^{+}\right)$be two weak entropy solutions to (1.1), with initial data $\rho_{0}, \sigma_{0} \in \mathbf{L}^{\infty}\left(I, \mathbb{R}^{+}\right)$and boundary data $\rho_{a}, \rho_{b}, \sigma_{a}, \sigma_{b} \in \mathbf{L}^{\infty}\left(\mathbb{R}^{+} ; \mathbb{R}^{+}\right)$ respectively. Then the following estimate holds:

$$
\|\rho(T, \cdot)-\sigma(T, \cdot)\|_{\mathbf{L}^{\mathbf{1}}(I)} \leq e^{\mathcal{S} T}\left[\left\|\rho_{0}-\sigma_{0}\right\|_{\mathbf{L}^{\mathbf{1}}(I)}+\left(L+\mathcal{S}^{\prime}\right)\left(\left\|\rho_{a}-\sigma_{a}\right\|_{\mathbf{L}^{\mathbf{1}}([0, T])}+\left\|\rho_{b}-\sigma_{b}\right\|_{\mathbf{L}^{\mathbf{1}}([0, T])}\right)\right],
$$

where the constants $\mathcal{S}, \mathcal{S}^{\prime}$ are defined by (3.10).

The above result can be easily generalized to unbounded domains $I=] a, b[$, with $a=-\infty$ or $b=+\infty$, under the assumption that the initial datum also belongs to $\mathbf{L}^{\mathbf{1}}(I)$. 


\section{Existence of weak entropy solutions}

The proof of existence is based on the following strategy: we construct a sequence of approximate solutions using a finite volume algorithm, we prove the convergence of a subsequence and, finally, we show that the limit is indeed a weak entropy solution in the sense of Definition 1 . The procedure follows closely $[1,5]$.

Let us fix a space grid in $[a, b]$ of size $\Delta x=(b-a) / N, N \in \mathbb{N}$, and choose a time step $\Delta t$ (satisfying some stability conditions which will be detailed later). We introduce the usual notation

$$
t^{n}=n \Delta t, n \in \mathbb{N} ; \quad x_{j}=a+\left(j-\frac{1}{2}\right) \Delta x, \quad x_{j+1 / 2}=a+j \Delta x, \quad j=1, \ldots, N ; \quad \lambda=\frac{\Delta t}{\Delta x} .
$$

Throughout, an initial datum $\rho_{0} \in\left(\mathbf{L}^{\infty} \cap \mathrm{BV}\right)(\mathbb{R} ; \mathbb{R})$ is fixed and we denote

$$
\rho_{j}^{0}=\frac{1}{\Delta x} \int_{x_{j-1 / 2}}^{x_{j+1 / 2}} \rho_{0}(x) d x d y \quad \text { for } j=1, \ldots, N .
$$

We define a piecewise constant approximate solution $\rho_{\Delta}$ to (1.1) as

$$
\rho_{\Delta}(t, x)=\rho_{j}^{n} \quad \text { for } \quad\left\{\begin{array}{l}
t \in\left[t^{n}, t^{n+1}[,\right. \\
x \in\left[x_{j-1 / 2}, x_{j+1 / 2}[,\right.
\end{array} \quad \text { where } \quad \begin{array}{l}
n \in \mathbb{N}, \\
j=1, \ldots, N,
\end{array}\right.
$$

through the following adapted Lax-Friedrichs scheme

$$
\rho_{j}^{n+1}=\rho_{j}^{n}-\lambda\left[F_{j+1 / 2}^{n}\left(\rho_{j}^{n}, \rho_{j+1}^{n}\right)-F_{j-1 / 2}^{n}\left(\rho_{j-1}^{n}, \rho_{j}^{n}\right)\right],
$$

where

$$
F_{j+1 / 2}^{n}\left(\rho_{j}^{n}, \rho_{j+1}^{n}\right):=\frac{1}{2}\left[f\left(t^{n}, x_{j}, \rho_{j}^{n}, R_{j}^{n}\right)+f\left(t^{n}, x_{j+1}, \rho_{j+1}^{n}, R_{j+1}^{n}\right)+\alpha\left(\rho_{j}^{n}-\rho_{j+1}^{n}\right)\right]
$$

is the numerical flux (for some $\alpha \in \mathbb{R}, \alpha>0$ ) and

$$
R_{j}^{n}:=\Delta x \sum_{k \in \mathbb{Z}} \eta\left(x_{j-k}\right) \rho_{k}^{n}, \quad j=1, \ldots, N,
$$

are the quadrature formulae approximating the convolution terms. Remark that, due to the boundedness of the domain $] a, b[$, we can set

$$
\begin{aligned}
R_{j}^{n} & :=\Delta x \sum_{k=1}^{N} \eta\left(x_{j-k}\right) \rho_{k}^{n}+\rho_{a}^{n} \Delta x \sum_{k \leq 0} \eta\left(x_{j-k}\right)+\rho_{b}^{n} \Delta x \sum_{k>N} \eta\left(x_{j-k}\right) \\
& =\Delta x \sum_{k=1}^{N} \eta\left(x_{j-k}\right) \rho_{k}^{n}+\rho_{a}^{n} \Delta x \sum_{k \geq j} \eta\left(x_{k}\right)+\rho_{b}^{n} \Delta x \sum_{k<j-N} \eta\left(x_{k}\right) .
\end{aligned}
$$

The proof of the convergence of approximate solutions is divided in several steps, which are intended to show that the sequence verifies the hypotheses of Helly's compactness theorem. 


\subsection{Positivity}

The following lemma ensures the positivity of approximate solutions corresponding to positive initial and boundary data.

Lemma 1 Let $\rho_{0} \in \mathbf{L}^{\infty}\left(I ; \mathbb{R}^{+}\right)$and $\rho_{a}, \rho_{b} \in \mathbf{L}^{\infty}\left(\mathbb{R}^{+} ; \mathbb{R}^{+}\right)$. Moreover, assume that

$$
\alpha \geq L, \quad \lambda \leq \frac{1}{3} \min \left\{\frac{1}{\alpha}, \frac{1}{L(1+\Delta x)}\right\} .
$$

Then $\rho_{\Delta}(t, x) \geq 0$ for all $x \in I, t>0$.

Proof. We rearrange $(2.1)$ as

$$
\begin{aligned}
\rho_{j}^{n+1} & =\rho_{j}^{n}-\lambda\left[F_{j+1 / 2}^{n}\left(\rho_{j}^{n}, \rho_{j+1}^{n}\right) \pm F_{j+1 / 2}^{n}\left(\rho_{j}^{n}, \rho_{j}^{n}\right) \pm F_{j-1 / 2}^{n}\left(\rho_{j}^{n}, \rho_{j}^{n}\right)-F_{j-1 / 2}^{n}\left(\rho_{j-1}^{n}, \rho_{j}^{n}\right)\right] \\
& =\left(1-\alpha_{j}^{n}-\beta_{j}^{n}\right) \rho_{j}^{n}+\alpha_{j}^{n} \rho_{j-1}^{n}+\beta_{j}^{n} \rho_{j+1}^{n}-\lambda\left(F_{j+1 / 2}^{n}\left(\rho_{j}^{n}, \rho_{j}^{n}\right)-F_{j-1 / 2}^{n}\left(\rho_{j}^{n}, \rho_{j}^{n}\right)\right),
\end{aligned}
$$

where, for $j \in\{1, \cdots, N\}$,

$$
\alpha_{j}^{n}:= \begin{cases}\lambda \frac{F_{j-1 / 2}^{n}\left(\rho_{j}^{n}, \rho_{j}^{n}\right)-F_{j-1 / 2}^{n}\left(\rho_{j-1}^{n}, \rho_{j}^{n}\right)}{\rho_{j}^{n}-\rho_{j-1}^{n}} & \text { if } \rho_{j}^{n} \neq \rho_{j-1}^{n}, \\ 0 & \text { if } \rho_{j}^{n}=\rho_{j-1}^{n},\end{cases}
$$

and

$$
\beta_{j}^{n}:= \begin{cases}-\lambda \frac{F_{j+1 / 2}^{n}\left(\rho_{j}^{n}, \rho_{j+1}^{n}\right)-F_{j+1 / 2}^{n}\left(\rho_{j}^{n}, \rho_{j}^{n}\right)}{\rho_{j+1}^{n}-\rho_{j}^{n}} & \text { if } \rho_{j+1}^{n} \neq \rho_{j}^{n}, \\ 0 & \text { if } \rho_{j+1}^{n}=\rho_{j}^{n} .\end{cases}
$$

We consider the following estimates:

$$
\begin{aligned}
& \left|F_{j+1 / 2}^{n}\left(\rho_{j}^{n}, \rho_{j}^{n}\right)-F_{j-1 / 2}^{n}\left(\rho_{j}^{n}, \rho_{j}^{n}\right)\right|= \\
= & \frac{1}{2}\left|f\left(t^{n}, x_{j+1}, \rho_{j}^{n}, R_{j+1}^{n}\right)-f\left(t^{n}, x_{j-1}, \rho_{j}^{n}, R_{j-1}^{n}\right)\right| \\
= & \frac{1}{2}\left|f\left(t^{n}, x_{j+1}, \rho_{j}^{n}, R_{j+1}^{n}\right) \pm f\left(t^{n}, x_{j-1}, \rho_{j}^{n}, R_{j+1}^{n}\right)-f\left(t^{n}, x_{j-1}, \rho_{j}^{n}, R_{j-1}^{n}\right)\right| \\
\leq & \frac{1}{2}\left|f\left(t^{n}, x_{j+1}, \rho_{j}^{n}, R_{j+1}^{n}\right)-f\left(t^{n}, x_{j-1}, \rho_{j}^{n}, R_{j+1}^{n}\right)\right| \\
& +\frac{1}{2}\left|f\left(t^{n}, x_{j-1}, \rho_{j}^{n}, R_{j+1}^{n}\right)\right|+\frac{1}{2}\left|f\left(t^{n}, x_{j-1}, \rho_{j}^{n}, R_{j-1}^{n}\right)\right| \\
\leq & L\left|\rho_{j}^{n}\right| \Delta x+\frac{1}{2}\left|f\left(t^{n}, x_{j-1}, \rho_{j}^{n}, R_{j+1}^{n}\right)-f\left(t^{n}, x_{j-1}, 0, R_{j+1}^{n}\right)\right| \\
& +\frac{1}{2}\left|f\left(t^{n}, x_{j-1}, \rho_{j}^{n}, R_{j-1}^{n}\right)-f\left(t^{n}, x_{j-1}, 0, R_{j-1}^{n}\right)\right| \\
\leq & L\left|\rho_{j}^{n}\right|(1+\Delta x) .
\end{aligned}
$$

Moreover, we observe that, whenever $\rho_{j}^{n} \neq \rho_{j-1}^{n}$ and $\rho_{j+1}^{n} \neq \rho_{j}^{n}$,

$$
\alpha_{j}^{n}=\frac{\lambda}{2\left(\rho_{j}^{n}-\rho_{j-1}^{n}\right)}\left[f\left(t^{n}, x_{j-1}, \rho_{j}^{n}, R_{j-1}^{n}\right)-f\left(t^{n}, x_{j-1}, \rho_{j-1}^{n}, R_{j-1}^{n}\right)+\alpha\left(\rho_{j}^{n}-\rho_{j-1}^{n}\right)\right]
$$




$$
=\frac{\lambda}{2}\left(\partial_{\rho} f\left(t^{n}, x_{j-1}, \xi_{j-1 / 2}^{n}, R_{j-1}^{n}\right)+\alpha\right)
$$

and

$$
\begin{aligned}
\beta_{j}^{n} & =-\frac{\lambda}{2\left(\rho_{j+1}^{n}-\rho_{j}^{n}\right)}\left[f\left(t^{n}, x_{j+1}, \rho_{j+1}^{n}, R_{j+1}^{n}\right)+\alpha\left(\rho_{j}^{n}-\rho_{j+1}^{n}\right)-f\left(t^{n}, x_{j+1}, \rho_{j}^{n}, R_{j+1}^{n}\right)\right] \\
& =\frac{\lambda}{2}\left(\alpha-\partial_{\rho} f\left(t^{n}, x_{j+1}, \xi_{j+1 / 2}^{n}, R_{j+1}^{n}\right)\right),
\end{aligned}
$$

for some $\xi_{j-1 / 2}^{n} \in \mathcal{I}\left(\rho_{j-1}^{n}, \rho_{j}^{n}\right)$ and $\xi_{j+1 / 2}^{n} \in \mathcal{I}\left(\rho_{j}^{n}, \rho_{j+1}^{n}\right)$. Assuming that

$$
\alpha \geq L, \quad \lambda \alpha \leq \frac{1}{3}, \quad \lambda L(1+\Delta x) \leq \frac{1}{3}
$$

we get

$$
\alpha_{j}^{n}, \beta_{j}^{n} \in\left[0, \frac{1}{3}\right], \quad\left(1-\alpha_{j}^{n}-\beta_{j}^{n}\right) \in\left[\frac{1}{3}, 1\right], \quad \lambda\left(F_{j+1 / 2}^{n}\left(\rho_{j}^{n}, \rho_{j}^{n}\right)-F_{j-1 / 2}^{n}\left(\rho_{j}^{n}, \rho_{j}^{n}\right)\right) \leq \frac{1}{3}\left|\rho_{j}^{n}\right|,
$$

which allow us to recover the sought estimate

$$
\begin{aligned}
\rho_{j}^{n+1} & \geq\left(1-\alpha_{j}^{n}-\beta_{j}^{n}\right) \rho_{j}^{n}+\alpha_{j}^{n} \rho_{j-1}^{n}+\beta_{j}^{n} \rho_{j+1}^{n}-\frac{1}{3}\left|\rho_{j}^{n}\right|, \\
& \geq\left(\frac{2}{3}-\alpha_{j}^{n}-\beta_{j}^{n}\right) \rho_{j}^{n}+\alpha_{j}^{n} \rho_{j-1}^{n}+\beta_{j}^{n} \rho_{j+1}^{n} \\
& \geq 0 .
\end{aligned}
$$

\section{$2.2 \quad \mathrm{~L}^{1}$ bound}

Lemma 2 Let hypotheses (1.2) and conditions (2.3) hold. If $\rho_{0} \in \mathbf{L}^{\infty}\left(I ; \mathbb{R}^{+}\right)$and $\rho_{a}, \rho_{b} \in$ $\mathbf{L}^{\infty}\left(\mathbb{R}^{+} ; \mathbb{R}^{+}\right)$, then for all $T>0$

$$
\left\|\rho_{\Delta}(T, \cdot)\right\|_{\mathbf{L}^{1}(I)} \leq\left\|\rho_{0}\right\|_{\mathbf{L}^{1}(I)}+\alpha\left(\left\|\rho_{a}\right\|_{\mathbf{L}^{1}([0, T])}+\left\|\rho_{b}\right\|_{\mathbf{L}^{1}([0, T])}\right)=: \mathcal{C}_{1}(T) .
$$

Proof. Thanks to the positivity of the discrete solution, using the definition of the scheme, we compute

$$
\begin{aligned}
\left\|\rho^{n+1}\right\|_{L^{1}(a, b)} & =\Delta x \sum_{j=1}^{N} \rho_{j}^{n+1} \\
& =\Delta x \sum_{j=1}^{N}\left[\rho_{j}^{n}-\lambda\left(F_{j+1 / 2}^{n}\left(\rho_{j}^{n}, \rho_{j+1}^{n}\right)-F_{j-1 / 2}^{n}\left(\rho_{j-1}^{n}, \rho_{j}^{n}\right)\right)\right] \\
& =\Delta x \sum_{j=1}^{N} \rho_{j}^{n}-\lambda \Delta x\left(F_{N+1 / 2}^{n}\left(\rho_{N}^{n}, \rho_{b}^{n}\right)-F_{1 / 2}^{n}\left(\rho_{a}^{n}, \rho_{1}^{n}\right)\right) \\
& =\Delta x \sum_{j=1}^{N} \rho_{j}^{n}-\frac{\Delta t}{2}\left(f\left(t^{n}, x_{N}, \rho_{N}^{n}, R_{N}^{n}\right)+f\left(t^{n}, x_{N+1}, \rho_{b}^{n}, R_{N+1}^{n}\right)+\alpha\left(\rho_{N}^{n}-\rho_{b}^{n}\right)\right)
\end{aligned}
$$




$$
\begin{aligned}
& +\frac{\Delta t}{2}\left(f\left(t^{n}, x_{0}, \rho_{a}^{n}, R_{0}^{n}\right)+f\left(t^{n}, x_{1}, \rho_{1}^{n}, R_{1}^{n}\right)+\alpha\left(\rho_{a}^{n}-\rho_{1}^{n}\right)\right) \\
= & \Delta x \sum_{j=1}^{N} \rho_{j}^{n} \\
& -\frac{\Delta t}{2}\left(\partial_{\rho} f\left(t^{n}, x_{N}, \xi_{N, 0}^{n}, R_{N}^{n}\right) \rho_{N}^{n}+\partial_{\rho} f\left(t^{n}, x_{N+1}, \xi_{N+1,0}^{n}, R_{N+1}^{n}\right) \rho_{b}^{n}+\alpha\left(\rho_{N}^{n}-\rho_{b}^{n}\right)\right) \\
& +\frac{\Delta t}{2}\left(\partial_{\rho} f\left(t^{n}, x_{0}, \xi_{a, 0}^{n}, R_{0}^{n}\right) \rho_{a}^{n}+\partial_{\rho} f\left(t^{n}, x_{1}, \xi_{1}^{n}, R_{1}^{n}\right) \rho_{1}^{n}+\alpha\left(\rho_{a}^{n}-\rho_{1}^{n}\right)\right) \\
= & \Delta x \sum_{j=1}^{N} \rho_{j}^{n}+\frac{\Delta t}{2}\left(\partial_{\rho} f\left(t^{n}, x_{0}, \xi_{a, 0}^{n}, R_{0}^{n}\right)+\alpha\right) \rho_{a}^{n} \\
& +\frac{\Delta t}{2}\left(-\partial_{\rho} f\left(t^{n}, x_{N+1}, \xi_{N+1,0}^{n}, R_{N+1}^{n}\right)+\alpha\right) \rho_{b}^{n} \\
& +\frac{\Delta t}{2}\left(-\partial_{\rho} f\left(t^{n}, x_{N}, \xi_{N, 0}^{n}, R_{N}^{n}\right)-\alpha\right) \rho_{N}^{n} \\
& +\frac{\Delta t}{2}\left(\partial_{\rho} f\left(t^{n}, x_{1}, \xi_{1}^{n}, R_{1}^{n}\right)-\alpha\right) \rho_{1}^{n} .
\end{aligned}
$$

Being the last two coefficients in the previous estimate non positive, we can conclude that

$$
\Delta x \sum_{j=1}^{N} \rho_{j}^{n+1} \leq \Delta x \sum_{j=1}^{N} \rho_{j}^{n}+\alpha \Delta t\left(\rho_{a}^{n}+\rho_{b}^{n}\right),
$$

thus ending the proof.

\section{$2.3 \quad \mathrm{~L}^{\infty}$ bound}

Lemma 3 Let hypotheses (1.2) and conditions (2.3) hold. If $\rho_{0} \in \mathbf{L}^{\infty}\left(I ; \mathbb{R}^{+}\right)$and $\rho_{a}, \rho_{b} \in$ $\mathbf{L}^{\infty}\left(\mathbb{R}^{+} ; \mathbb{R}^{+}\right)$, then for all $T>0$

$$
\left\|\rho_{\Delta}(T, \cdot)\right\|_{\mathbf{L}^{\infty}(I)} \leq e^{\mathcal{L} T}\left\|\rho_{0}\right\|_{\mathbf{L}^{\infty}(I)}
$$

where $\mathcal{L}$ is given by $(2.12)$.

Proof. We observe that

$$
\begin{aligned}
& \left|R_{j+1}^{n}-R_{j-1}^{n}\right| \leq \\
\leq & \Delta x \rho_{a}^{n} \sum_{k \leq 0}\left|\eta\left(x_{j+1-k}\right)-\eta\left(x_{j-1-k}\right)\right|+\Delta x \sum_{k=1}^{N} \rho_{k}^{n}\left|\eta\left(x_{j+1-k}\right)-\eta\left(x_{j-1-k}\right)\right| \\
& +\Delta x \rho_{b}^{n} \sum_{k \geq N+1}\left|\eta\left(x_{j+1-k}\right)-\eta\left(x_{j-1-k}\right)\right| \\
= & \Delta x \rho_{a}^{n} \sum_{k \leq 0}\left|\int_{x_{j-1-k}}^{j+1-k} \eta^{\prime}(s) d s\right|+\Delta x \sum_{k=1}^{N} \rho_{k}^{n}\left|\int_{x_{j-1-k}}^{j+1-k} \eta^{\prime}(s) d s\right|+\Delta x \rho_{b}^{n} \sum_{k \leq 0}\left|\int_{x_{j-1-k}}^{j+1-k} \eta^{\prime}(s) d s\right| \\
\leq & 2 \Delta x^{2}\left\|\eta^{\prime}\right\|_{\mathbf{L}^{\infty}(\mathbb{R})}\left(\rho_{a}^{n}+\sum_{j=1}^{N} \rho_{j}^{n}+\rho_{b}^{n}\right)
\end{aligned}
$$




$$
\begin{aligned}
& \leq 2 \Delta x\left\|\eta^{\prime}\right\|_{\mathbf{L}^{\infty}(\mathbb{R})}\left(\left\|\rho_{0}\right\|_{\mathbf{L}^{1}(I)}+\alpha\left(\left\|\rho_{a}\right\|_{\mathbf{L}^{1}([0, T])}+\left\|\rho_{b}\right\|_{\mathbf{L}^{1}([0, T])}\right)\right) \\
& \leq 2 \mathcal{T} \Delta x
\end{aligned}
$$

where we have set

$$
\mathcal{T}:=\left\|\eta^{\prime}\right\|_{\mathbf{L}^{\infty}(\mathbb{R})}\left(\left\|\rho_{0}\right\|_{\mathbf{L}^{\mathbf{1}}(I)}+\alpha\left(\left\|\rho_{a}\right\|_{\mathbf{L}^{\mathbf{1}}([0, T])}+\left\|\rho_{b}\right\|_{\mathbf{L}^{\mathbf{1}}([0, T])}\right)\right)
$$

and for the latest bound we have applied Lemma 2 .

Proceding as in Lemma 1, we can rearrange (2.1) as

$$
\rho_{j}^{n+1}=\left(1-\alpha_{j}^{n}-\beta_{j}^{n}\right) \rho_{j}^{n}+\alpha_{j}^{n} \rho_{j-1}^{n}+\beta_{j}^{n} \rho_{j+1}^{n}-\lambda\left(F_{j+1 / 2}^{n}\left(\rho_{j}^{n}, \rho_{j}^{n}\right)-F_{j-1 / 2}^{n}\left(\rho_{j}^{n}, \rho_{j}^{n}\right)\right),
$$

where $\alpha_{j}^{n}$ and $\beta_{j}^{n}$ are as in (2.6) and (2.7) respectively. Using (2.10), we get

$$
\begin{aligned}
& \left|F_{j+1 / 2}^{n}\left(\rho_{j}^{n}, \rho_{j}^{n}\right)-F_{j-1 / 2}^{n}\left(\rho_{j}^{n}, \rho_{j}^{n}\right)\right|= \\
& =\frac{1}{2}\left|f\left(t^{n}, x_{j+1}, \rho_{j}^{n}, R_{j+1}^{n}\right)-f\left(t^{n}, x_{j-1}, \rho_{j}^{n}, R_{j-1}^{n}\right)\right| \\
& =\frac{1}{2}\left(\left|\partial_{x} f\left(t^{n}, \tilde{x}_{j}, \rho_{j}^{n}, \tilde{R}_{j}^{n}\right)\right|\left|x_{j+1}-x_{j-1}\right|+\left|\partial_{x} f\left(t^{n}, \tilde{x}_{j}, \rho_{j}^{n}, \tilde{R}_{j}^{n}\right)\right|\left|R_{j+1}^{n}-R_{j-1}^{n}\right|\right) \\
& \leq 2 C\left|\rho_{j}^{n}\right| \Delta x+C\left|\rho_{j}^{n}\right|\left|R_{j+1}^{n}-R_{j-1}^{n}\right| \\
& \leq 2 C \Delta x\left|\rho_{j}^{n}\right|(1+\mathcal{T}) .
\end{aligned}
$$

Thus,

$$
\begin{aligned}
\rho_{j}^{n+1} & \leq\left(1-\alpha_{j}^{n}-\beta_{j}^{n}\right) \rho_{j}^{n}+\alpha_{j}^{n} \rho_{j-1}^{n}+\beta_{j}^{n} \rho_{j+1}^{n}+\lambda\left|F_{j+1 / 2}^{n}\left(\rho_{j}^{n}, \rho_{j}^{n}\right)-F_{j-1 / 2}^{n}\left(\rho_{j}^{n}, \rho_{j}^{n}\right)\right| \\
& \leq\left(1-\alpha_{j}^{n}-\beta_{j}^{n}\right)\left\|\rho^{n}\right\|_{\mathbf{L}^{\infty}(I)}+\alpha_{j}^{n}\left\|\rho^{n}\right\|_{\mathbf{L}^{\infty}(I)}+\beta_{j}^{n}\left\|\rho^{n}\right\|_{\mathbf{L}^{\infty}(I)}+2 C \lambda \Delta x\left\|\rho^{n}\right\|_{\mathbf{L}^{\infty}(I)}(1+\mathcal{T}) \\
& =\left\|\rho^{n}\right\|_{\mathbf{L}^{\infty}(I)}(1+\Delta t \mathcal{L}) \\
& \leq e^{\mathcal{L} \Delta t}\left\|\rho^{n}\right\|_{\mathbf{L}^{\infty}(I)},
\end{aligned}
$$

for $j=1, \cdots, N$, being

$$
\mathcal{L}:=2 C\left(1+\left\|\eta^{\prime}\right\|_{\mathbf{L}^{\infty}(\mathbb{R})}\left(\left\|\rho_{0}\right\|_{\mathbf{L}^{1}(I)}+\alpha\left(\left\|\rho_{a}\right\|_{\mathbf{L}^{1}([0, T])}+\left\|\rho_{b}\right\|_{\mathbf{L}^{1}([0, T])}\right)\right)\right) .
$$

A standard iterative argument completes the proof.

\subsection{BV estimates}

Proposition 1 (BV estimate in space) Let hypotheses (1.2) and conditions (2.3) hold. If $\rho_{0} \in \mathbf{L}^{\infty}\left(I ; \mathbb{R}^{+}\right)$and $\rho_{a}, \rho_{b} \in \mathbf{L}^{\infty}\left(\mathbb{R}^{+} ; \mathbb{R}^{+}\right)$, then $\rho_{\Delta}$ satisfies the following Total Variation estimate

$$
\sum_{j=0}^{N}\left|\rho_{j+1}^{n}-\rho_{j}^{n}\right| \leq \mathcal{C}_{x}\left(t^{n}\right)
$$


for all $n \in \mathbb{N}$, where

$$
\mathcal{C}_{x}\left(t^{n}\right):=e^{\mathcal{K}_{1} t^{n}} \sum_{j=0}^{N}\left|\rho_{j+1}^{0}-\rho_{j}^{0}\right|+\frac{\mathcal{K}_{2}}{\mathcal{K}_{1}}\left(e^{\mathcal{K}_{1} t^{n}}-1\right)+\sum_{m=1}^{n}\left|\rho_{a}^{m}-\rho_{a}^{m-1}\right|+\sum_{m=1}^{n}\left|\rho_{b}^{m}-\rho_{b}^{m-1}\right|,
$$

with $\mathcal{K}_{1}$ and $\mathcal{K}_{2}$ positive constants defined in (2.18), (2.19), (2.24).

Proof. We consider separately the central and boundary terms.

For $j=1, \cdots, N-1, n \in \mathbb{N}$, we consider the difference

$$
\begin{aligned}
\rho_{j+1}^{n+1}-\rho_{j}^{n+1}= & \rho_{j+1}^{n}-\rho_{j}^{n}-\lambda\left[F_{j+3 / 2}^{n}\left(\rho_{j+1}^{n}, \rho_{j+2}^{n}\right)-F_{j-1 / 2}^{n}\left(\rho_{j-1}^{n}, \rho_{j}^{n}\right) \pm F_{j+1 / 2}^{n}\left(\rho_{j}^{n}, \rho_{j+1}^{n}\right)\right] \\
& \mp \lambda\left(F_{j+3 / 2}^{n}\left(\rho_{j}^{n}, \rho_{j+1}^{n}\right)+F_{j+1 / 2}^{n}\left(\rho_{j-1}^{n}, \rho_{j}^{n}\right)\right) \\
= & \rho_{j+1}^{n}-\rho_{j}^{n} \\
& -\lambda\left(F_{j+3 / 2}^{n}\left(\rho_{j+1}^{n}, \rho_{j+2}^{n}\right)-F_{j+1 / 2}^{n}\left(\rho_{j}^{n}, \rho_{j+1}^{n}\right)-F_{j+3 / 2}^{n}\left(\rho_{j}^{n}, \rho_{j+1}^{n}\right)+F_{j+1 / 2}^{n}\left(\rho_{j-1}^{n}, \rho_{j}^{n}\right)\right) \\
& -\lambda\left(F_{j+3 / 2}^{n}\left(\rho_{j}^{n}, \rho_{j+1}^{n}\right)-F_{j+1 / 2}^{n}\left(\rho_{j}^{n}, \rho_{j+1}^{n}\right)+F_{j-1 / 2}^{n}\left(\rho_{j-1}^{n}, \rho_{j}^{n}\right)-F_{j+1 / 2}^{n}\left(\rho_{j-1}^{n}, \rho_{j}^{n}\right)\right) \\
= & \mathcal{A}_{j}^{n}-\lambda \mathcal{B}_{j}^{n},
\end{aligned}
$$

where we have set

$$
\begin{aligned}
& \mathcal{A}_{j}^{n}:=\rho_{j+1}^{n}-\rho_{j}^{n}-\lambda\left(F_{j+3 / 2}^{n}\left(\rho_{j+1}^{n}, \rho_{j+2}^{n}\right)-F_{j+1 / 2}^{n}\left(\rho_{j}^{n}, \rho_{j+1}^{n}\right)-F_{j+3 / 2}^{n}\left(\rho_{j}^{n}, \rho_{j+1}^{n}\right)+F_{j+1 / 2}^{n}\left(\rho_{j-1}^{n}, \rho_{j}^{n}\right)\right), \\
& \mathcal{B}_{j}^{n}:=F_{j+3 / 2}^{n}\left(\rho_{j}^{n}, \rho_{j+1}^{n}\right)-F_{j+1 / 2}^{n}\left(\rho_{j}^{n}, \rho_{j+1}^{n}\right)+F_{j-1 / 2}^{n}\left(\rho_{j-1}^{n}, \rho_{j}^{n}\right)-F_{j+1 / 2}^{n}\left(\rho_{j-1}^{n}, \rho_{j}^{n}\right) .
\end{aligned}
$$

Concerning the first term $\mathcal{A}_{j}^{n}$ and recalling (2.4) and (2.5), after suitable rearrangements we get

$$
\begin{aligned}
\mathcal{A}_{j}^{n}= & \left(\rho_{j+1}^{n}-\rho_{j}^{n}\right) \\
& \times\left[1+\lambda \frac{F_{j+1 / 2}^{n}\left(\rho_{j}^{n}, \rho_{j+1}^{n}\right)-F_{j+1 / 2}^{n}\left(\rho_{j}^{n}, \rho_{j}^{n}\right)}{\rho_{j+1}^{n}-\rho_{j}^{n}}-\lambda \frac{F_{j+3 / 2}^{n}\left(\rho_{j+1}^{n}, \rho_{j+1}^{n}\right)-F_{j+3 / 2}^{n}\left(\rho_{j}^{n}, \rho_{j+1}^{n}\right)}{\rho_{j+1}^{n}-\rho_{j}^{n}}\right] \\
& +\left(\rho_{j+2}^{n}-\rho_{j+1}^{n}\right)\left(-\lambda \frac{F_{j+3 / 2}^{n}\left(\rho_{j+1}^{n}, \rho_{j+2}^{n}\right)-F_{j+3 / 2}^{n}\left(\rho_{j+1}^{n}, \rho_{j+1}^{n}\right)}{\rho_{j+2}^{n}-\rho_{j+1}^{n}}\right) \\
& +\left(\rho_{j}^{n}-\rho_{j-1}^{n}\right)\left(\lambda \frac{F_{j+1 / 2}^{n}\left(\rho_{j}^{n}, \rho_{j}^{n}\right)-F_{j+1 / 2}^{n}\left(\rho_{j-1}^{n}, \rho_{j}^{n}\right)}{\rho_{j}^{n}-\rho_{j-1}^{n}}\right) \\
= & \left(1-\beta_{j}^{n}-\gamma_{j+1}^{n}\right)\left(\rho_{j+1}^{n}-\rho_{j}^{n}\right)+\beta_{j+1}^{n}\left(\rho_{j+2}^{n}-\rho_{j+1}^{n}\right)+\gamma_{j}^{n}\left(\rho_{j}^{n}-\rho_{j-1}^{n}\right),
\end{aligned}
$$

where

$$
\gamma_{j}^{n}:=\lambda \frac{F_{j+1 / 2}^{n}\left(\rho_{j}^{n}, \rho_{j}^{n}\right)-F_{j+1 / 2}^{n}\left(\rho_{j-1}^{n}, \rho_{j}^{n}\right)}{\rho_{j}^{n}-\rho_{j-1}^{n}},
$$

and the bounds $\gamma_{j}^{n} \in[0,1 / 3]$ can be proved exactly as it has been done for $\alpha_{j}^{n}$, thanks to $(2.3)$. From (2.15) we recover

$$
\sum_{j=1}^{N-1}\left|\mathcal{A}_{j}^{n}\right| \leq \sum_{j=1}^{N-1}\left[\left(1-\beta_{j}^{n}-\gamma_{j+1}^{n}\right)\left|\rho_{j+1}^{n}-\rho_{j}^{n}\right|+\beta_{j+1}^{n}\left|\rho_{j+2}^{n}-\rho_{j+1}^{n}\right|+\gamma_{j}^{n}\left|\rho_{j}^{n}-\rho_{j-1}^{n}\right|\right]
$$




$$
=\sum_{j=1}^{N-1}\left|\rho_{j+1}^{n}-\rho_{j}^{n}\right|-\beta_{1}^{n}\left|\rho_{2}^{n}-\rho_{1}^{n}\right|+\gamma_{1}^{n}\left|\rho_{1}^{n}-\rho_{a}^{n}\right|+\beta_{N}^{n}\left|\rho_{b}^{n}-\rho_{N}^{n}\right|-\gamma_{N}^{n}\left|\rho_{N}^{n}-\rho_{N-1}^{n}\right| .
$$

We now focus on the term $\mathcal{B}_{j}^{n}$ in $(2.14)$ :

$$
\begin{aligned}
\mathcal{B}_{j}^{n}= & \frac{1}{2}\left(f\left(t^{n}, x_{j+2}, \rho_{j+1}^{n}, R_{j+2}^{n}\right)-f\left(t^{n}, x_{j+1}, \rho_{j+1}^{n}, R_{j+1}^{n}\right)\right) \\
& -\frac{1}{2}\left(f\left(t^{n}, x_{j}, \rho_{j-1}^{n}, R_{j}^{n}\right)-f\left(t^{n}, x_{j-1}, \rho_{j-1}^{n}, R_{j-1}^{n}\right)\right) \\
= & \left(\partial_{x} f\left(t^{n}, \tilde{x}_{j+3 / 2}, \rho_{j+1}^{n}, \tilde{R}_{j+3 / 2}^{n}\right) \Delta x+\partial_{R} f\left(t^{n}, \tilde{x}_{j+3 / 2}, \rho_{j+1}^{n}, \tilde{R}_{j+3 / 2}^{n}\right)\left(R_{j+2}^{n}-R_{j+1}^{n}\right)\right) \\
& -\frac{1}{2}\left(\partial_{x} f\left(t^{n}, \tilde{x}_{j-1 / 2}, \rho_{j-1}^{n}, \tilde{R}_{j-1 / 2}^{n}\right) \Delta x+\partial_{R} f\left(t^{n}, \tilde{x}_{j-1 / 2}, \rho_{j-1}^{n}, \tilde{R}_{j-1 / 2}^{n}\right)\left(R_{j}^{n}-R_{j-1}^{n}\right)\right) \\
= & \frac{\Delta x}{2}\left(\partial_{x x}^{2} f\left(t^{n}, \hat{x}_{j}, \hat{\rho}_{j}^{n}, \hat{R}_{j}^{n}\right)\left(\tilde{x}_{j+3 / 2}-\tilde{x}_{j-1 / 2}\right)+\partial_{\rho x}^{2} f\left(t^{n}, \hat{x}_{j}, \hat{\rho}_{j}^{n}, \hat{R}_{j}^{n}\right)\left(\rho_{j+1}^{n}-\rho_{j-1}^{n}\right)\right. \\
& \left.+\partial_{R x}^{2} f\left(t^{n}, \hat{x}_{j}, \hat{\rho}_{j}^{n}, \hat{R}_{j}^{n}\right)\left(\tilde{R}_{j+3 / 2}^{n}-\tilde{R}_{j-1 / 2}^{n}\right)\right) \\
& +\frac{1}{2}\left(\partial_{x R}^{2} f\left(t^{n}, \bar{x}_{j}, \bar{\rho}_{j}^{n}, \bar{R}_{j}^{n}\right) \check{R}_{j+1 / 2}^{n}\left(\tilde{x}_{j+3 / 2}-\tilde{x}_{j-1 / 2}\right)+\partial_{\rho R}^{2} f\left(t^{n}, \bar{x}_{j}, \bar{\rho}_{j}^{n}, \bar{R}_{j}^{n}\right) \check{R}_{j+1 / 2}^{n}\left(\rho_{j+1}^{n}-\rho_{j-1}^{n}\right)\right. \\
& +\partial_{R R}^{2} f\left(t^{n}, \bar{x}_{j}, \bar{\rho}_{j}^{n}, \bar{R}_{j}^{n}\right) \check{R}_{j+1 / 2}^{n}\left(\tilde{R}_{j+3 / 2}^{n}-\tilde{R}_{j-1 / 2}^{n}\right) \\
& \left.+\partial_{R} f\left(t^{n}, \bar{x}_{j}, \bar{\rho}_{j}^{n}, \bar{R}_{j}^{n}\right)\left(R_{j+2}^{n}-R_{j+1}^{n}-R_{j}^{n}+R_{j-1}^{n}\right)\right),
\end{aligned}
$$

with $\tilde{x}_{j-1 / 2} \in\left(x_{j-1}, x_{j}\right), \hat{\rho}_{j}^{n}, \bar{\rho}_{j}^{n} \in \mathcal{I}\left(\rho_{j-1}^{n}, \rho_{j+1}^{n}\right), \tilde{R}_{j-1 / 2}^{n} \in \mathcal{I}\left(R_{j-1}^{n}, R_{j}^{n}\right), \hat{R}_{j}^{n}, \bar{R}_{j}^{n} \in \mathcal{I}\left(\tilde{R}_{j-1 / 2}^{n}, \tilde{R}_{j+3 / 2}^{n}\right)$, $\hat{x}_{j}, \bar{x}_{j} \in \mathcal{I}\left(\tilde{x}_{j-1 / 2} \tilde{x}_{j+3 / 2}\right), \check{R}_{j+1 / 2}^{n} \in \mathcal{I}\left(R_{j}^{n}-R_{j-1}^{n}, R_{j+2}^{n}-R_{j+1}^{n}\right)$.

Notice that as in (2.10) we can estimate

$$
\begin{aligned}
\left|R_{j+2}^{n}-R_{j+1}^{n}\right| & \leq \mathcal{T} \Delta x, \\
\left|R_{j}^{n}-R_{j-1}^{n}\right| & \leq \mathcal{T} \Delta x, \\
\left|R_{j+2}^{n}-R_{j}^{n}\right| & \leq 2 \mathcal{T} \Delta x .
\end{aligned}
$$

Moreover, by their very definition,

$$
\begin{aligned}
\left|\tilde{R}_{j+3 / 2}^{n}-\tilde{R}_{j-1 / 2}^{n}\right| & =\left|\lambda_{j+3 / 2}^{n} R_{j+2}^{n}+\left(1-\lambda_{j+3 / 2}^{n}\right) R_{j+1}^{n}-\mu_{j-1 / 2}^{n} R_{j-1}^{n}-\left(1-\mu_{j-1 / 2}^{n}\right) R_{j}^{n}\right| \\
& \leq\left|R_{j+2}^{n}-R_{j+1}^{n}\right|+\left|R_{j}^{n}-R_{j-1}^{n}\right|+\left|R_{j+1}^{n}-R_{j}^{n}\right| \\
& \leq 3 \mathcal{T} \Delta x,
\end{aligned}
$$

and

$$
\begin{aligned}
\left|\check{R}_{j+1 / 2}^{n}\right| & =\left|\delta_{j+1 / 2}^{n}\left(R_{j}^{n}-R_{j-1}^{n}\right)+\left(1-\delta_{j+1 / 2}^{n}\right)\left(R_{j+2}^{n}-R_{j+1}^{n}\right)\right| \\
& \leq \delta_{j+1 / 2}^{n}\left|R_{j}^{n}-R_{j-1}^{n}\right|+\left(1-\delta_{j+1 / 2}^{n}\right)\left|R_{j+2}^{n}-R_{j+1}^{n}\right| \\
& \leq \mathcal{T} \Delta x
\end{aligned}
$$

for some $\delta_{j+1 / 2}^{n}, \lambda_{j+3 / 2}^{n}, \mu_{j-1 / 2}^{n} \in[0,1]$. Finally,

$$
\left|\hat{\rho}_{j}^{n}\right|=\left|\hat{\epsilon}_{j}^{n} \rho_{j-1}^{n}+\left(1-\hat{\epsilon}_{j}^{n}\right) \rho_{j+1}^{n}\right| \leq \hat{\epsilon}_{j}^{n}\left|\rho_{j-1}^{n}\right|+\left(1-\hat{\epsilon}_{j}^{n}\right)\left|\rho_{j+1}^{n}\right|,
$$




$$
\left|\bar{\rho}_{j}^{n}\right|=\left|\bar{\epsilon}_{j}^{n} \rho_{j-1}^{n}+\left(1-\bar{\epsilon}_{j}^{n}\right) \rho_{j+1}^{n}\right| \leq \bar{\epsilon}_{j}^{n}\left|\rho_{j-1}^{n}\right|+\left(1-\bar{\epsilon}_{j}^{n}\right)\left|\rho_{j+1}^{n}\right|,
$$

with $\hat{\epsilon}_{j}^{n}, \bar{\epsilon}_{j}^{n} \in[0,1]$. Therefore

$$
\begin{aligned}
\left|\mathcal{B}_{j}^{n}\right| \leq & \frac{3}{2} C(\Delta x)^{2}\left|\hat{\rho}_{j}^{n}\right|+\frac{1}{2} \Delta x\left\|\partial_{\rho x}^{2} f\right\|_{\mathbf{L}^{\infty}}\left|\rho_{j+1}^{n}-\rho_{j-1}^{n}\right| \\
& +\frac{3}{2} C \mathcal{T}(\Delta x)^{2}\left|\hat{\rho}_{j}^{n}\right|+\frac{3}{2} C \mathcal{T}(\Delta x)^{2}\left|\bar{\rho}_{j}^{n}\right|+\frac{1}{2} \mathcal{T}\left\|\partial_{\rho R}^{2} f\right\|_{\mathbf{L} \infty} \Delta x\left|\rho_{j+1}^{n}-\rho_{j-1}^{n}\right| \\
& +\frac{3}{2} C \mathcal{T}^{2}(\Delta x)^{2}\left|\bar{\rho}_{j}^{n}\right|+C \mathcal{T}(\Delta x)^{2}\left|\bar{\rho}_{j}^{n}\right| \\
\leq & \frac{1}{2} \Delta x\left|\rho_{j+1}^{n}-\rho_{j-1}^{n}\right|\left(\left\|\partial_{\rho x}^{2} f\right\|_{\mathbf{L}^{\infty}(\mathbb{R})}+\mathcal{T}\left\|\partial_{\rho R}^{2} f\right\|_{\mathbf{L}^{\infty}}\right) \\
& +\frac{3}{2} C(1+\mathcal{T})(\Delta x)^{2}\left|\hat{\rho}_{j}^{n}\right|+\frac{1}{2} C \mathcal{T}(5+3 \mathcal{T})(\Delta x)^{2}\left|\bar{\rho}_{j}^{n}\right|
\end{aligned}
$$

Thus

$$
\begin{aligned}
\sum_{j=1}^{N-1} \lambda\left|\mathcal{B}_{j}^{n}\right| \leq & \frac{1}{2} \Delta t\left(\left\|\partial_{\rho x}^{2} f\right\|_{\mathbf{L}^{\infty}}+\mathcal{T}\left\|\partial_{\rho R}^{2} f\right\|_{\mathbf{L}^{\infty}}\right) \sum_{j=1}^{N-1}\left|\rho_{j+1}^{n}-\rho_{j-1}^{n}\right| \\
& +\frac{1}{2} C \Delta t(3(1+\mathcal{T})+\mathcal{T}(5+3 \mathcal{T})) \Delta x \sum_{j=1}^{N-1}\left[\left(\hat{\epsilon}_{j}^{n}+\bar{\epsilon}_{j}^{n}\right)\left|\rho_{j-1}^{n}\right|+\left(2-\hat{\epsilon}_{j}^{n}-\bar{\epsilon}_{j}^{n}\right)\left|\rho_{j+1}^{n}\right|\right] \\
\leq & \mathcal{K}_{1} \Delta t \sum_{j=0}^{N}\left|\rho_{j+1}^{n}-\rho_{j}^{n}\right|+\widetilde{\mathcal{K}}_{2} \Delta t \Delta x \sum_{j=1}^{N}\left|\rho_{j+1}^{n}\right|
\end{aligned}
$$

where we have set

$$
\begin{aligned}
\mathcal{K}_{1} & =\left\|\partial_{\rho x}^{2} f\right\|_{\mathbf{L}^{\infty}}+\mathcal{T}\left\|\partial_{\rho R}^{2} f\right\|_{\mathbf{L}^{\infty}} \\
\widetilde{\mathcal{K}}_{2} & =C\left(3+8 \mathcal{T}+3 \mathcal{T}^{2}\right) .
\end{aligned}
$$

Collecting (2.16) and (2.17), we conclude that

$$
\begin{aligned}
\sum_{j=1}^{N}\left|\rho_{j+1}^{n+1}-\rho_{j}^{n+1}\right| & \leq \sum_{j=1}^{N}\left|\mathcal{A}_{j}^{n}\right|+\lambda \sum_{j=1}^{N}\left|\mathcal{B}_{j}^{n}\right| \\
& \leq\left(1+\mathcal{K}_{1} \Delta t\right) \sum_{j=1}^{N}\left|\rho_{j+1}^{n}-\rho_{j}^{n}\right|+\widetilde{\mathcal{K}}_{2} \Delta t \Delta x \sum_{j=1}^{N}\left|\rho_{j+1}^{n}\right|+\frac{1}{2} \mathcal{K}_{1} \Delta t\left|\rho_{1}^{n}-\rho_{a}^{n}\right| \\
& \leq\left(1+\mathcal{K}_{1} \Delta t\right) \sum_{j=1}^{N}\left|\rho_{j+1}^{n}-\rho_{j}^{n}\right|+\widetilde{\mathcal{K}}_{2} \mathcal{C}_{1}\left(t^{n}\right) \Delta t+\frac{1}{2} \mathcal{K}_{1} \Delta t\left|\rho_{1}^{n}-\rho_{a}^{n}\right| .
\end{aligned}
$$

We now take into account the boundary terms. From the definition of the scheme we know that

$$
\begin{aligned}
\rho_{1}^{n+1}-\rho_{a}^{n+1} & =\left(1-\alpha_{1}^{n}-\beta_{1}^{n}\right) \rho_{1}^{n}+\alpha_{1}^{n} \rho_{a}^{n}+\beta_{1}^{n} \rho_{2}^{n}-\lambda\left(F_{3 / 2}^{n}\left(\rho_{1}^{n}, \rho_{1}^{n}\right)-F_{1 / 2}^{n}\left(\rho_{1}^{n}, \rho_{1}^{n}\right)\right)-\rho_{a}^{n+1} \pm \rho_{a}^{n} \\
& =\beta_{1}^{n}\left(\rho_{2}^{n}-\rho_{1}^{n}\right)+\left(1-\alpha_{1}^{n}\right)\left(\rho_{1}^{n}-\rho_{a}^{n}\right)+\left(\rho_{a}^{n}-\rho_{a}^{n+1}\right)
\end{aligned}
$$




$$
\begin{aligned}
& -\lambda\left(F_{3 / 2}^{n}\left(\rho_{1}^{n}, \rho_{1}^{n}\right)-F_{1 / 2}^{n}\left(\rho_{1}^{n}-\rho_{1}^{n}\right)\right) \\
= & \beta_{1}^{n}\left(\rho_{2}^{n}-\rho_{1}^{n}\right)+\left(1-\gamma_{1}^{n}\right)\left(\rho_{1}^{n}-\rho_{a}^{n}\right)+\left(\rho_{a}^{n}-\rho_{a}^{n+1}\right) \\
& -\lambda\left(F_{3 / 2}^{n}\left(\rho_{a}^{n}, \rho_{1}^{n}\right)-F_{1 / 2}^{n}\left(\rho_{a}^{n}, \rho_{1}^{n}\right)\right) .
\end{aligned}
$$

Indeed, by definition of $\alpha_{1}^{n}$ and of $\gamma_{1}^{n}$ we get

$$
\begin{aligned}
\alpha_{1}^{n} & \left(\rho_{1}^{n}-\rho_{a}^{n}\right)+\lambda\left(F_{3 / 2}^{n}\left(\rho_{1}^{n}, \rho_{1}^{n}\right)-F_{1 / 2}^{n}\left(\rho_{1}^{n}, \rho_{1}^{n}\right)\right)= \\
& =\lambda\left(F_{3 / 2}^{n}\left(\rho_{1}^{n}, \rho_{1}^{n}\right)-F_{1 / 2}^{n}\left(\rho_{a}^{n}, \rho_{1}^{n}\right) \pm F_{3 / 2}^{n}\left(\rho_{a}^{n}, \rho_{1}^{n}\right)\right) \\
& =\lambda \frac{F_{3 / 2}^{n}\left(\rho_{1}^{n}, \rho_{1}^{n}\right)-F_{3 / 2}^{n}\left(\rho_{a}^{n}, \rho_{1}^{n}\right)}{\rho_{1}^{n}-\rho_{a}^{n}}\left(\rho_{1}^{n}-\rho_{a}^{n}\right)+\lambda\left(F_{3 / 2}^{n}\left(\rho_{a}^{n}, \rho_{1}^{n}\right)-F_{1 / 2}^{n}\left(\rho_{a}^{n}, \rho_{1}^{n}\right)\right) \\
& =\gamma_{1}^{n}\left(\rho_{1}^{n}-\rho_{a}^{n}\right)+\lambda\left(F_{3 / 2}^{n}\left(\rho_{a}^{n}, \rho_{1}^{n}\right)-F_{1 / 2}^{n}\left(\rho_{a}^{n}, \rho_{1}^{n}\right)\right) .
\end{aligned}
$$

Since

$$
\begin{aligned}
\lambda & \left(F_{3 / 2}^{n}\left(\rho_{a}^{n}, \rho_{1}^{n}\right)-F_{1 / 2}^{n}\left(\rho_{a}^{n}, \rho_{1}^{n}\right)\right)= \\
= & \frac{\lambda}{2}\left(f\left(t^{n}, x_{1}, \rho_{a}^{n}, R_{1}^{n}\right)-f\left(t^{n}, x_{0}, \rho_{a}^{n}, R_{0}^{n}\right)\right)+\frac{\lambda}{2}\left(f\left(t^{n}, x_{2}, \rho_{1}^{n}, R_{2}^{n}\right)-f\left(t^{n}, x_{1}, \rho_{1}^{n}, R_{1}^{n}\right)\right) \\
= & \frac{\lambda}{2}\left(\partial_{x} f\left(t^{n}, \tilde{x}_{1 / 2}, \rho_{a}^{n}, \tilde{R}_{1 / 2}^{n}\right) \Delta x+\partial_{R} f\left(t^{n}, \tilde{x}_{1 / 2}, \rho_{a}^{n}, \tilde{R}_{1 / 2}^{n}\right)\left(R_{1}^{n}-R_{0}^{n}\right)\right) \\
& +\frac{\lambda}{2}\left(\partial_{x} f\left(t^{n}, \tilde{x}_{3 / 2}, \rho_{1}^{n}, \tilde{R}_{3 / 2}^{n}\right) \Delta x+\partial_{R} f\left(t^{n}, \tilde{x}_{3 / 2}, \rho_{1}^{n}, \tilde{R}_{3 / 2}^{n}\right)\left(R_{2}^{n}-R_{1}^{n}\right)\right)
\end{aligned}
$$

(where we used obvious notations for $\tilde{x}_{1 / 2}, \tilde{x}_{3 / 2}, \tilde{R}_{1 / 2}^{n}$ and $\tilde{R}_{3 / 2}^{n}$ ), we can conclude that

$$
\lambda\left|F_{3 / 2}^{n}\left(\rho_{a}^{n}, \rho_{1}^{n}\right)-F_{1 / 2}^{n}\left(\rho_{a}^{n}, \rho_{1}^{n}\right)\right| \leq \Delta t \frac{C}{2}(1+\mathcal{T})\left(\left|\rho_{a}^{n}\right|+\left|\rho_{1}^{n}\right|\right) .
$$

Thus, because of the positivity of the coefficients involved, we get

$$
\begin{aligned}
\left|\rho_{1}^{n+1}-\rho_{a}^{n+1}\right| & \leq \beta_{1}^{n}\left|\rho_{2}^{n}-\rho_{1}^{n}\right|+\left(1-\gamma_{1}^{n}\right)\left|\rho_{1}^{n}-\rho_{a}^{n}\right|+\left|\rho_{a}^{n}-\rho_{a}^{n+1}\right| \\
& +\Delta t \frac{C}{2}(1+\mathcal{T})\left(\left|\rho_{a}^{n}\right|+\left|\rho_{1}^{n}\right|\right) .
\end{aligned}
$$

Concerning the right boundary data, we have

$$
\begin{aligned}
\rho_{N}^{n+1}-\rho_{b}^{n+1}= & \left(1-\alpha_{N}^{n}-\beta_{N}^{n}\right) \rho_{N}^{n}+\alpha_{N}^{n} \rho_{N-1}^{n}+\beta_{N}^{n} \rho_{b}^{n}-\lambda\left(F_{N+1 / 2}^{n}\left(\rho_{N}^{n}, \rho_{N}^{n}\right)-F_{N-1 / 2}^{n}\left(\rho_{N}^{n}, \rho_{N}^{n}\right)\right) \\
& -\rho_{b}^{n+1} \pm \rho_{b}^{n} \\
= & -\alpha_{N}^{n}\left(\rho_{N}^{n}-\rho_{N-1}^{n}\right)+\left(1-\beta_{N}^{n}\right)\left(\rho_{N}^{n}-\rho_{b}^{n}\right)+\left(\rho_{b}^{n}-\rho_{b}^{n+1}\right) \\
& +\alpha_{N}^{n}\left(\rho_{N}^{n}-\rho_{N-1}^{n}\right)-\gamma_{N}^{n}\left(\rho_{N}^{n}-\rho_{N-1}^{n}\right) \\
& -\lambda\left(F_{N+1 / 2}^{n}\left(\rho_{N-1}^{n}, \rho_{N}^{n}\right)-F_{N-1 / 2}^{n}\left(\rho_{N-1}^{n}, \rho_{N}^{n}\right)\right) \\
= & \left(1-\beta_{N}^{n}\right)\left(\rho_{N}^{n}-\rho_{b}^{n}\right)+\left(\rho_{b}^{n}-\rho_{b}^{n+1}\right)-\gamma_{N}^{n}\left(\rho_{N}^{n}-\rho_{N-1}^{n}\right) \\
& -\lambda\left(F_{N+1 / 2}^{n}\left(\rho_{N-1}^{n}, \rho_{N}^{n}\right)-F_{N-1 / 2}^{n}\left(\rho_{N-1}^{n}, \rho_{N}^{n}\right)\right) .
\end{aligned}
$$


We can justify the above equalities as follows. Taking into account of the expressions of $\alpha_{N}^{n}$ and of $\gamma_{N}^{n}$, we can rearrange

$$
\begin{aligned}
-\lambda & \left(F_{N+1 / 2}^{n}\left(\rho_{N}^{n}, \rho_{N}^{n}\right)-F_{N-1 / 2}^{n}\left(\rho_{N}^{n}, \rho_{N}^{n}\right)\right)= \\
= & -\lambda\left(F_{N+1 / 2}^{n}\left(\rho_{N}^{n}, \rho_{N}^{n}\right)-F_{N-1 / 2}^{n}\left(\rho_{N}^{n}, \rho_{N}^{n}\right) \pm F_{N-1 / 2}^{n}\left(\rho_{N-1}^{n}, \rho_{N}^{n}\right) \pm F_{N+1 / 2}^{n}\left(\rho_{N-1}^{n}, \rho_{N}^{n}\right)\right) \\
= & -\lambda\left(F_{N-1 / 2}^{n}\left(\rho_{N-1}^{n}, \rho_{N}^{n}\right)-F_{N-1 / 2}^{n}\left(\rho_{N}^{n}, \rho_{N}^{n}\right)\right)-\lambda\left(F_{N+1 / 2}^{n}\left(\rho_{N}^{n}, \rho_{N}^{n}\right)-F_{N+1 / 2}^{n}\left(\rho_{N-1}^{n}, \rho_{N}^{n}\right)\right) \\
& -\lambda\left(F_{N+1 / 2}^{n}\left(\rho_{N-1}^{n}, \rho_{N}^{n}\right)-F_{N-1 / 2}^{n}\left(\rho_{N-1}^{n}, \rho_{N}^{n}\right)\right) \\
= & \alpha_{N}^{n}\left(\rho_{N}^{n}-\rho_{N-1}^{n}\right)-\gamma_{N}^{n}\left(\rho_{N}^{n}-\rho_{N-1}^{n}\right)-\lambda\left(F_{N+1 / 2}^{n}\left(\rho_{N-1}^{n}, \rho_{N}^{n}\right)-F_{N-1 / 2}^{n}\left(\rho_{N-1}^{n}, \rho_{N}^{n}\right)\right) .
\end{aligned}
$$

Let us estimate the last term in (2.22):

$$
\begin{aligned}
& \lambda\left|F_{N+1 / 2}^{n}\left(\rho_{N-1}^{n}, \rho_{N}^{n}\right)-F_{N-1 / 2}^{n}\left(\rho_{N-1}^{n}, \rho_{N}^{n}\right)\right|= \\
& =\frac{\lambda}{2}\left|f\left(t^{n}, x_{N}, \rho_{N-1}^{n}, R_{N}^{n}\right)-f\left(t^{n}, x_{N-1}, \rho_{N-1}^{n}, R_{N-1}^{n}\right)+f\left(t^{n}, x_{N+1}, \rho_{N}^{n}, R_{N+1}^{n}\right)-f\left(t^{n}, x_{N}, \rho_{N}^{n}, R_{N}^{n}\right)\right| \\
& =\frac{\lambda}{2} \mid \partial_{x} f\left(t^{n}, \tilde{x}_{N-1 / 2}, \rho_{N-1}^{n}, \tilde{R}_{N-1 / 2}^{n}\right) \Delta x+\partial_{R} f\left(t^{n}, \tilde{x}_{N-1 / 2}, \rho_{N-1}^{n}, \tilde{R}_{N-1 / 2}^{n}\right)\left(R_{N}^{n}-R_{N-1}^{n}\right) \\
& \quad \quad \quad \partial_{x} f\left(t^{n}, \tilde{x}_{N+1 / 2}, \rho_{N}^{n}, \tilde{R}_{N+1 / 2}^{n}\right) \Delta x+\partial_{R} f\left(t^{n}, \tilde{x}_{N+1 / 2}, \rho_{N}^{n}, \tilde{R}_{N+1 / 2}^{n}\right)\left(R_{N+1}^{n}-R_{N}^{n}\right) \mid \\
& \leq \Delta t \frac{C}{2}(1+\mathcal{T})\left(\left|\rho_{N-1}^{n}\right|+\left|\rho_{N}^{n}\right|\right) .
\end{aligned}
$$

Therefore we get

$$
\begin{aligned}
\left|\rho_{N}^{n+1}-\rho_{b}^{n+1}\right| & \leq\left(1-\beta_{N}^{n}\right)\left|\rho_{N}^{n}-\rho_{b}^{n}\right|+\gamma_{N}^{n}\left|\rho_{N}^{n}-\rho_{N-1}^{n}\right|+\left|\rho_{b}^{n}-\rho_{b}^{n+1}\right| \\
& +\Delta t \frac{C}{2}(1+\mathcal{T})\left(\left|\rho_{N-1}^{n}\right|+\left|\rho_{N}^{n}\right|\right) .
\end{aligned}
$$

Collecting estimates $(2.20),(2.21)$ and (2.23), we obtain

$$
\begin{aligned}
& \sum_{j=0}^{N}\left|\rho_{j+1}^{n+1}-\rho_{j}^{n+1}\right| \\
& =\left|\rho_{1}^{n+1}-\rho_{a}^{n+1}\right|+\left|\rho_{b}^{n+1}-\rho_{N}^{n+1}\right|+\sum_{j=1}^{N-1}\left|\mathcal{A}_{j}^{n}-\lambda \mathcal{B}_{j}^{n}\right| \\
& \leq \beta_{1}^{n}\left|\rho_{2}^{n}-\rho_{1}^{n}\right|+\left(1-\gamma_{1}^{n}\right)\left|\rho_{1}^{n}-\rho_{a}^{n}\right|+\left|\rho_{a}^{n}-\rho_{a}^{n+1}\right|+\Delta t \frac{C}{2}(1+\mathcal{T})\left(\left|\rho_{a}^{n}\right|+\left|\rho_{1}^{n}\right|\right) \\
& \quad+\left(1-\beta_{N}^{n}\right)\left|\rho_{b}^{n}-\rho_{N}^{n}\right|+\gamma_{N}^{n}\left|\rho_{N}^{n}-\rho_{N-1}^{n}\right|+\left|\rho_{b}^{n}-\rho_{b}^{n+1}\right|+\Delta t \frac{C}{2}(1+\mathcal{T})\left(\left|\rho_{N-1}^{n}\right|+\left|\rho_{N}^{n}\right|\right) \\
& \quad+\sum_{j=1}^{N-1}\left|\rho_{j+1}^{n}-\rho_{j}^{n}\right|-\beta_{1}^{n}\left|\rho_{2}^{n}-\rho_{1}^{n}\right|+\gamma_{1}^{n}\left|\rho_{1}^{n}-\rho_{a}^{n}\right|+\beta_{N}^{n}\left|\rho_{b}^{n}-\rho_{N}^{n}\right|-\gamma_{N}^{n}\left|\rho_{N}^{n}-\rho_{N-1}^{n}\right| \\
& \quad+\lambda \sum_{j=1}^{N-1}\left|\mathcal{B}_{j}^{n}\right|
\end{aligned}
$$




$$
\begin{aligned}
\leq & \left|\rho_{a}^{n}-\rho_{a}^{n+1}\right|+\left|\rho_{b}^{n}-\rho_{b}^{n+1}\right|+\sum_{j=0}^{N}\left|\rho_{j+1}^{n}-\rho_{j}^{n}\right| \\
& +\Delta t \frac{C}{2}(1+\mathcal{T})\left(\left|\rho_{a}^{n}\right|+\left|\rho_{1}^{n}\right|+\left|\rho_{N-1}^{n}\right|+\left|\rho_{N}^{n}\right|\right) \\
& +\mathcal{K}_{1} \Delta t \sum_{j=0}^{N}\left|\rho_{j+1}^{n}-\rho_{j}^{n}\right|+\widetilde{\mathcal{K}}_{2} \Delta t \Delta x \sum_{j=1}^{N}\left|\rho_{j+1}^{n}\right| \\
\leq & \left|\rho_{a}^{n}-\rho_{a}^{n+1}\right|+\left|\rho_{b}^{n}-\rho_{b}^{n+1}\right|+\left(1+\mathcal{K}_{1} \Delta t\right) \sum_{j=0}^{N}\left|\rho_{j+1}^{n}-\rho_{j}^{n}\right|+\widetilde{\mathcal{K}}_{2} \mathcal{C}_{1}\left(t^{n}\right) \Delta t \\
& +C(1+\mathcal{T})\left(\frac{3}{2} e^{\mathcal{L} t^{n}}\left\|\rho_{0}\right\|_{\mathbf{L}^{\infty}(I)}+\frac{1}{2}\left\|\rho_{a}\right\|_{\mathbf{L}^{\infty}([0, T])}\right) \Delta t .
\end{aligned}
$$

Setting

$$
\mathcal{K}_{2}:=\widetilde{\mathcal{K}}_{2} \mathcal{C}_{1}\left(t^{n}\right)+C(1+\mathcal{T})\left(\frac{3}{2} e^{\mathcal{L} t^{n}}\left\|\rho_{0}\right\|_{\mathbf{L}^{\infty}(I)}+\frac{1}{2}\left\|\rho_{a}\right\|_{\mathbf{L}^{\infty}[0, T]}\right)
$$

we deduce from the previous estimate

$$
\begin{aligned}
\sum_{j=0}^{N}\left|\rho_{j+1}^{n}-\rho_{j}^{n}\right| \leq & \left(1+\mathcal{K}_{1} \Delta t\right)^{t^{n} / \Delta t} \sum_{j=0}^{N}\left|\rho_{j+1}^{0}-\rho_{j}^{0}\right|+\mathcal{K}_{2} \frac{\left(1+\mathcal{K}_{1} \Delta t\right)^{t^{n} / \Delta t}-1}{\mathcal{K}_{1}} \\
& +\sum_{m=1}^{n}\left|\rho_{a}^{m}-\rho_{a}^{m-1}\right|+\sum_{m=1}^{n}\left|\rho_{b}^{m}-\rho_{b}^{m-1}\right| \\
\leq & e^{\mathcal{K}_{1} t^{n}} \sum_{j=0}^{N}\left|\rho_{j+1}^{0}-\rho_{j}^{0}\right|+\frac{\mathcal{K}_{2}}{\mathcal{K}_{1}}\left(e^{\mathcal{K}_{1} t^{n}}-1\right)+\sum_{m=1}^{n}\left|\rho_{a}^{m}-\rho_{a}^{m-1}\right|+\sum_{m=1}^{n}\left|\rho_{b}^{m}-\rho_{b}^{m-1}\right|
\end{aligned}
$$

thus concluding the proof.

Corollary 2 (BV estimate in space and time) Let hypotheses (1.2) and conditions (2.3) hold. If $\rho_{0} \in \mathbf{L}^{\infty}\left(I ; \mathbb{R}^{+}\right)$and $\rho_{a}, \rho_{b} \in \mathbf{L}^{\infty}\left(\mathbb{R}^{+} ; \mathbb{R}^{+}\right)$, then $\rho_{\Delta}$ satisfies the following Total Variation estimate in space and time

$$
\sum_{m=0}^{n-1} \sum_{j=0}^{N} \Delta t\left|\rho_{j+1}^{m}-\rho_{j}^{m}\right|+\sum_{m=0}^{n-1} \sum_{j=0}^{N} \Delta x\left|\rho_{j}^{m+1}-\rho_{j}^{m}\right| \leq \mathcal{C}_{x t}(n \Delta t),
$$

with $\mathcal{C}_{x t}(n \Delta t)$ given by $(2.30)$

Proof. The spatial BV estimate (2.13) yields

$$
\sum_{m=0}^{n-1} \sum_{j=0}^{N} \Delta t\left|\rho_{j+1}^{m}-\rho_{j}^{m}\right| \leq n \Delta t \mathcal{C}_{x}(n \Delta t) .
$$

In order to bound the second term in (2.25), we make use of the definition of the numerical scheme (2.1), (2.2). In fact, by (1.2) and (2.10) we have the following estimate

$$
\left|\rho_{j}^{m+1}-\rho_{j}^{m}\right| \leq \frac{\lambda \alpha}{2}\left(\left|\rho_{j}^{m}-\rho_{j-1}^{m}\right|+\left|\rho_{j+1}^{m}-\rho_{j}^{m}\right|\right)
$$




$$
\begin{aligned}
& +\frac{\lambda}{2}\left[2\left|\partial_{x} f\left(t^{m}, \tilde{x}_{j}, \xi_{j}^{m}, \tilde{R}_{j}^{m}\right)\right| \Delta x+\left|\partial_{\rho} f\left(t^{m}, \tilde{x}_{j}, \xi_{j}^{m}, \tilde{R}_{j}^{m}\right)\left(\rho_{j+1}^{m}-\rho_{j-1}^{m}\right)\right|\right. \\
& \left.+\left|\partial_{R} f\left(t^{m}, \tilde{x}_{j}, \xi_{j}^{m}, \tilde{R}_{j}^{m}\right)\left(R_{j+1}^{m}-R_{j-1}^{m}\right)\right|\right] \\
\leq & \frac{\lambda}{2}(\alpha+L)\left(\left|\rho_{j}^{m}-\rho_{j-1}^{m}\right|+\left|\rho_{j+1}^{m}-\rho_{j}^{m}\right|\right) \\
& +C \Delta t\left|\epsilon \rho_{j+1}^{m}+(1-\epsilon) \rho_{j-1}^{m}\right|+\mathcal{T} \Delta t\left|\epsilon \rho_{j+1}^{m}+(1-\epsilon) \rho_{j-1}^{m}\right| \\
\leq & \frac{\lambda}{2}(\alpha+L)\left(\left|\rho_{j}^{m}-\rho_{j-1}^{m}\right|+\left|\rho_{j+1}^{m}-\rho_{j}^{m}\right|\right)+\Delta t(C+\mathcal{T})\left(\epsilon\left|\rho_{j+1}^{m}\right|+(1-\epsilon)\left|\rho_{j-1}^{m}\right|\right)
\end{aligned}
$$

for $j=1, \cdots, N-1$, where $\xi_{j}^{m}=\epsilon \rho_{j+1}^{m}+(1-\epsilon) \rho_{j-1}^{m} \in \mathcal{I}\left(\rho_{j-1}^{m}, \rho_{j-1}^{m}\right), \epsilon \in[0,1], \tilde{x}_{j} \in\left[x_{j-1}, x_{j+1}\right]$ and $\tilde{R}_{j}^{m} \in \mathcal{I}\left(R_{j-1}^{m}, R_{j+1}^{m}\right)$. Therefore,

$$
\begin{aligned}
\sum_{j=0}^{N} \Delta x\left|\rho_{j}^{m+1}-\rho_{j}^{m}\right|= & \Delta x\left(\left|\rho_{a}^{m+1}-\rho_{a}^{m}\right|+\left|\rho_{b}^{m+1}-\rho_{b}^{m}\right|\right)+\sum_{j=1}^{N-1} \Delta x\left|\rho_{j}^{m+1}-\rho_{j}^{m}\right| \\
\leq & \Delta x\left(\left|\rho_{a}^{m+1}-\rho_{a}^{m}\right|+\left|\rho_{b}^{m+1}-\rho_{b}^{m}\right|\right) \\
& +\Delta t(\alpha+L) \sum_{j=0}^{N-1}\left|\rho_{j+1}^{m}-\rho_{j}^{m}\right|+2 \Delta x \Delta t(C+\mathcal{T}) \sum_{j=0}^{N}\left|\rho_{j}^{m}\right| \\
\leq & \Delta x\left(\left|\rho_{a}^{m+1}-\rho_{a}^{m}\right|+\left|\rho_{b}^{m+1}-\rho_{b}^{m}\right|\right) \\
& +\Delta t(\alpha+L) \mathcal{C}_{x}(m \Delta t)+2 \Delta x \Delta t(C+\mathcal{T}) \mathcal{C}_{1}(m \Delta t),
\end{aligned}
$$

due to estimates (2.13) and (2.8). In particular,

$$
\sum_{j=0}^{N} \Delta x\left|\rho_{j}^{m+1}-\rho_{j}^{m}\right| \leq \Delta t \mathcal{C}_{t}(m \Delta t),
$$

where

$$
\mathcal{C}_{t}(m \Delta t):=\lambda\left(\left|\rho_{a}^{m+1}-\rho_{a}^{m}\right|+\left|\rho_{b}^{m+1}-\rho_{b}^{m}\right|\right)+(\alpha+L) \mathcal{C}_{x}(m \Delta t)+2 \Delta x(C+\mathcal{T}) \mathcal{C}_{1}(m \Delta t),
$$

which allows to derive the $\mathbf{L}^{1}$ Lipschitz continuity in time (1.12).

Summing over $=0, \cdots, n-1$, we get

$$
\begin{aligned}
\sum_{m=0}^{n-1} \sum_{j=0}^{N} \Delta x\left|\rho_{j}^{m+1}-\rho_{j}^{m}\right| \leq & \Delta x \sum_{m=0}^{n-1}\left(\left|\rho_{a}^{m+1}-\rho_{a}^{m}\right|+\left|\rho_{b}^{m+1}-\rho_{b}^{m}\right|\right) \\
& +n \Delta t(\alpha+L) \mathcal{C}_{x}(n \Delta t)+2 n \Delta t \Delta x(C+\mathcal{T}) \mathcal{C}_{1}(n \Delta t) .
\end{aligned}
$$

Summing (2.26) and (2.29) we get (2.25) with

$$
\mathcal{C}_{x t}(n \Delta t):=n \Delta t(\alpha+L+1) \mathcal{C}_{x}(n \Delta t)
$$




$$
+\Delta x\left[2 \Delta t(C+\mathcal{T}) \mathcal{C}_{1}(n \Delta t)+\sum_{m=0}^{n-1}\left(\left|\rho_{a}^{m+1}-\rho_{a}^{m}\right|+\left|\rho_{b}^{m+1}-\rho_{b}^{m}\right|\right)\right],
$$

thus completing the proof.

\subsection{Discrete entropy inequalities}

We adopt the following notation

$$
\begin{aligned}
F_{j+1 / 2}^{n}(u, v) & :=\frac{1}{2}\left(f\left(t^{n}, x_{j}, u, R_{j}^{n}\right)+f\left(t^{n}, x_{j+1}, v, R_{j+1}^{n}\right)+\alpha(u-v)\right), \\
H_{j}(u, v, z) & :=v-\lambda\left(F_{j+1 / 2}^{n}(v, z)-F_{j-1 / 2}^{n}(u, v)\right), \\
G_{j+1 / 2}^{\kappa} & :=F_{j+1 / 2}^{n}(u \wedge \kappa, v \wedge \kappa)-F_{j+1 / 2}^{n}(\kappa, \kappa) .
\end{aligned}
$$

The approximate solution $\rho_{\Delta}$ satisfies the following inequalities.

Lemma 4 Under the hypotheses (1.2) and the conditions (2.3), the following discrete entropy inequalities hold for $j=1, \cdots, N, n \in \mathbb{N}, \kappa \in \mathbb{R}$ :

$$
\begin{aligned}
& \left(\rho_{j}^{n+1}-\kappa\right)^{+}-\left(\rho_{j}^{n}-\kappa\right)^{+}+\lambda\left(G_{j+1 / 2}^{\kappa}\left(\rho_{j}^{n}, \rho_{j+1}^{n}\right)-G_{j-1 / 2}^{\kappa}\left(\rho_{j-1}^{n}, \rho_{j}^{n}\right)\right) \\
& \quad+\frac{\lambda}{2} \operatorname{sgn}^{+}\left(\rho_{j}^{n+1}-\kappa\right)\left(f\left(t^{n}, x_{j+1}, \kappa, R_{j+1}^{n}\right)-f\left(t^{n}, x_{j-1}, \kappa, R_{j-1}^{n}\right)\right) \leq 0 .
\end{aligned}
$$

Proof. Let us consider the map $(u, v, z) \mapsto H_{j}(u, v, z)$. By (2.3), it holds

$$
\begin{aligned}
& \frac{\partial H}{\partial u}(u, v, z)=-\frac{\lambda}{2}\left(\partial_{\rho} f\left(t^{n}, x_{j-1}, u, R_{j-1}^{n}\right)-\alpha\right) \geq 0, \\
& \frac{\partial H}{\partial v}(u, v, z)=1-\frac{\alpha \lambda}{2} \geq 0, \\
& \frac{\partial H}{\partial z}(u, v, z)=-\frac{\lambda}{2}\left(\partial_{\rho} f\left(t^{n}, x_{j+1}, z, R_{j+1}^{n}\right)-\alpha\right) \geq 0 .
\end{aligned}
$$

Notice that

$$
H_{j}(\kappa, \kappa, \kappa)=\kappa-\frac{\lambda}{2}\left(f\left(t^{n}, x_{j+1}, \kappa, R_{j+1}^{n}\right)-f\left(t^{n}, x_{j-1}, \kappa, R_{j-1}^{n}\right)\right) .
$$

For $\kappa \in \mathbb{R}, j=1, \cdots, N$, noticing that $\rho_{j}^{n+1}=H_{j}\left(\rho_{j-1}^{n}, \rho_{j}^{n}, \rho_{j+1}^{n}\right)$ and using the monotonicity above, we get

$$
\begin{aligned}
& H_{j}\left(\rho_{j-1}^{n} \wedge \kappa, \rho_{j}^{n} \wedge \kappa, \rho_{j+1}^{n} \wedge \kappa\right)-H_{j}(\kappa, \kappa, \kappa) \geq \\
& \quad \geq H_{j}\left(\rho_{j-1}^{n}, \rho_{j}^{n}, \rho_{j+1}^{n}\right) \wedge H_{j}(\kappa, \kappa, \kappa)-H_{j}(\kappa, \kappa, \kappa) \\
& \quad=\left(H_{j}\left(\rho_{j-1}^{n}, \rho_{j}^{n}, \rho_{j+1}^{n}\right)-H_{j}(\kappa, \kappa, \kappa)\right)^{+} \\
& \quad=\left(\rho_{j}^{n+1}-\kappa+\frac{\lambda}{2}\left(f\left(t^{n}, x_{j+1}, \kappa, R_{j+1}^{n}\right)-f\left(t^{n}, x_{j-1}, \kappa, R_{j-1}^{n}\right)\right)\right)^{+} .
\end{aligned}
$$

On the other hand we obtain

$$
H_{j}\left(\rho_{j-1}^{n} \wedge \kappa, \rho_{j}^{n} \wedge \kappa, \rho_{j+1}^{n} \wedge \kappa\right)-H_{j}(\kappa, \kappa, \kappa)=
$$




$$
\begin{aligned}
= & \left(\rho_{j}^{n}-\kappa\right)^{+} \\
& -\lambda\left(F_{j+1 / 2}^{n}\left(\rho_{j}^{n} \wedge \kappa, \rho_{j+1}^{n} \wedge \kappa\right)-F_{j-1 / 2}^{n}\left(\rho_{j-1}^{n} \wedge \kappa, \rho_{j}^{n} \wedge \kappa\right)-F_{j+1 / 2}^{n}(\kappa, \kappa)+F_{j-1 / 2}^{n}(\kappa, \kappa)\right) \\
= & \left(\rho_{j}^{n}-\kappa\right)^{+}-\lambda\left(G_{j+1 / 2}^{\kappa}\left(\rho_{j}^{n}, \rho_{j+1}^{n}\right)-G_{j-1 / 2}^{\kappa}\left(\rho_{j-1}^{n}, \rho_{j}^{n}\right)\right) .
\end{aligned}
$$

Thus,

$$
\begin{aligned}
& \left(\rho_{j}^{n}-\kappa\right)^{+}-\lambda\left(G_{j+1 / 2}^{\kappa}\left(\rho_{j}^{n}, \rho_{j+1}^{n}\right)-G_{j-1 / 2}^{\kappa}\left(\rho_{j-1}^{n}, \rho_{j}^{n}\right)\right) \geq \\
\geq & \left(\rho_{j}^{n+1}-\kappa+\frac{\lambda}{2}\left(f\left(t^{n}, x_{j+1}, \kappa, R_{j+1}^{n}\right)-f\left(t^{n}, x_{j-1}, \kappa, R_{j-1}^{n}\right)\right)\right)^{+} \\
= & \operatorname{sgn}^{+}\left(\rho_{j}^{n+1}-\kappa+\frac{\lambda}{2}\left(f\left(t^{n}, x_{j+1}, \kappa, R_{j+1}^{n}\right)-f\left(t^{n}, x_{j-1}, \kappa, R_{j-1}^{n}\right)\right)\right) \\
& \times\left(\rho_{j}^{n+1}-\kappa+\frac{\lambda}{2}\left(f\left(t^{n}, x_{j+1}, \kappa, R_{j+1}^{n}\right)-f\left(t^{n}, x_{j-1}, \kappa, R_{j-1}^{n}\right)\right)\right) \\
\geq & \operatorname{sgn}^{+}\left(\rho_{j}^{n+1}-\kappa\right)\left(\rho_{j}^{n+1}-\kappa+\frac{\lambda}{2}\left(f\left(t^{n}, x_{j+1}, \kappa, R_{j+1}^{n}\right)-f\left(t^{n}, x_{j-1}, \kappa, R_{j-1}^{n}\right)\right)\right) \\
= & \left(\rho_{j}^{n+1}-\kappa\right)^{+}+\frac{\lambda}{2} \operatorname{sgn}^{+}\left(\rho_{j}^{n+1}-\kappa\right)\left(f\left(t^{n}, x_{j+1}, \kappa, R_{j+1}^{n}\right)-f\left(t^{n}, x_{j-1}, \kappa, R_{j-1}^{n}\right)\right),
\end{aligned}
$$

which proves (2.31).

\subsection{Convergence towards a weak entropy solution}

The estimates given by Lemmas 3 and Corollary 2 allow to apply Helly's compactness theorem, ensuring the existence of a subsequence, still denoted $\left\{\rho_{\Delta}\right\}$ converging to a function $\rho \in \mathbf{L}^{\infty}([0, T] \times$ $I$ ) in the $\mathbf{L}^{\mathbf{1}}$-norm, for all $T>0$ (see for example [16, Section 5.3.5]). We need now to prove that the limit of approximate solutions is indeed a weak entropy solution, in the sense of Definition 1.

Lemma 5 Let hypotheses (1.2) and conditions (2.3) hold. If $\rho_{0} \in\left(\mathbf{L}^{\infty} \cap B V\right)\left(I ; \mathbb{R}^{+}\right)$and $\rho_{a}, \rho_{b} \in$ $\left(\mathbf{L}^{\infty} \cap B V\right)\left(\mathbb{R}^{+} ; \mathbb{R}^{+}\right)$, then the piecewise constant approximate solutions $\rho_{\Delta}$ resulting from the adapted Lax-Friedrichs scheme (2.1) converge, as $\Delta x \searrow 0$, towards a weak entropy solution of the initial boundary value problem (1.1).

Proof. We follow closely [27]. Adding and subtracting $G_{j+1 / 2}^{\kappa}\left(\rho_{j}^{n}, \rho_{j}^{n}\right)$, we rearrange (2.31) as

$$
\begin{aligned}
0 & \geq\left(\rho_{j}^{n+1}-\kappa\right)^{+}-\left(\rho_{j}^{n}-\kappa\right)^{+}+\lambda\left(G_{j+1 / 2}^{\kappa}\left(\rho_{j}^{n}, \rho_{j+1}^{n}\right)-G_{j+1 / 2}^{\kappa}\left(\rho_{j}^{n}, \rho_{j}^{n}\right)\right) \\
& +\lambda\left(G_{j+1 / 2}^{\kappa}\left(\rho_{j}^{n}, \rho_{j}^{n}\right)-G_{j-1 / 2}^{\kappa}\left(\rho_{j-1}^{n}, \rho_{j}^{n}\right)\right) \\
& +\frac{\lambda}{2} \operatorname{sgn}^{+}\left(\rho_{j}^{n+1}-\kappa\right)\left(f\left(t^{n}, x_{j+1}, \kappa, R_{j+1}^{n}\right)-f\left(t^{n}, x_{j-1}, \kappa, R_{j-1}^{n}\right)\right) .
\end{aligned}
$$

Let $\varphi \in C_{c}^{1}\left([0, T) \times[a, b], \mathbb{R}^{+}\right)$for some $T>0$. Multiplying $(2.32)$ by $\Delta x \varphi\left(t^{n}, x_{j}\right) \geq 0$, and summing over $j=1, \cdots, N, n \in \mathbb{N}$, we get the inequality

$0 \geq \Delta x \sum_{n=0}^{+\infty} \sum_{j=1}^{N}\left(\eta_{\kappa}^{+}\left(\rho_{j}^{n+1}\right)-\eta_{\kappa}^{+}\left(\rho_{j}^{n}\right)\right) \varphi\left(t^{n}, x_{j}\right)$ 


$$
\begin{aligned}
& +\Delta t \sum_{n=0}^{+\infty} \sum_{j=1}^{N}\left(\left(G_{j+1 / 2}^{\kappa}\left(\rho_{j}^{n}, \rho_{j+1}^{n}\right)-G_{j+1 / 2}^{\kappa}\left(\rho_{j}^{n}, \rho_{j}^{n}\right)\right)-\left(G_{j-1 / 2}^{\kappa}\left(\rho_{j-1}^{n}, \rho_{j}^{n}\right)-G_{j+1 / 2}^{\kappa}\left(\rho_{j}^{n}, \rho_{j}^{n}\right)\right)\right) \varphi\left(t^{n}, x_{j}\right) \\
& +\frac{\Delta t}{2} \sum_{n=0}^{+\infty} \sum_{j=1}^{N} \operatorname{sgn}^{+}\left(\rho_{j}^{n+1}-\kappa\right)\left(f\left(t^{n}, x_{j+1}, \kappa, R_{j+1}^{n}\right)-f\left(t^{n}, x_{j-1}, \kappa, R_{j-1}^{n}\right)\right) \varphi\left(t^{n}, x_{j}\right) .
\end{aligned}
$$

Summing by parts in (2.33) we obtain

$$
\begin{aligned}
T_{1} & :=\Delta x \sum_{n=0}^{+\infty} \sum_{j=1}^{N}\left(\eta_{\kappa}^{+}\left(\rho^{n+1}\right)-\eta_{\kappa}^{+}\left(\rho_{j}^{n}\right)\right) \varphi\left(t^{n}, x_{j}\right) \\
& =-\Delta x \sum_{j=1}^{N} \varphi\left(0, x_{j}\right) \eta_{\kappa}^{+}\left(\rho_{j}^{0}\right)-\Delta x \Delta t \sum_{n=1}^{+\infty} \sum_{j=1}^{N} \eta_{\kappa}^{+}\left(\rho_{j}^{n}\right) \frac{\varphi\left(t^{n}, x_{j}\right)-\varphi\left(t^{n-1}, x_{j}\right)}{\Delta t} \\
& \longrightarrow \Delta x \searrow^{+}-\int_{a}^{b} \varphi(0, x) \rho_{0}(x) d x-\int_{0}^{+\infty} \int_{a}^{b} \partial_{t} \varphi(t, x) \eta_{\kappa}^{+}(\rho(t, x)) d x d t
\end{aligned}
$$

and

$$
\begin{aligned}
T_{3} & :=\frac{\Delta t}{2} \sum_{n=0}^{+\infty} \sum_{j=1}^{N} \operatorname{sgn}^{ \pm}\left(\rho_{j}^{n+1}-\kappa\right)\left(f\left(t^{n}, x_{j+1}, \kappa, R_{j+1}^{n}\right)-f\left(t^{n}, x_{j-1}, \kappa, R_{j-1}^{n}\right)\right) \varphi\left(t^{n}, x_{j}\right) \\
& =\Delta t \Delta x \sum_{n=0}^{+\infty} \sum_{j=1}^{N} \operatorname{sgn}^{+}\left(\rho_{j}^{n+1}-\kappa\right) \frac{f\left(t^{n}, x_{j+1}, \kappa, R_{j+1}^{n}\right)-f\left(t^{n}, x_{j-1}, \kappa, R_{j-1}^{n}\right)}{2 \Delta x} \\
& \longrightarrow \Delta x \searrow 0^{+} \int_{0}^{+\infty} \int_{a}^{b} \operatorname{sgn}^{+}\left(\rho_{j}^{n+1}-\kappa\right) \partial_{x} f(t, x, \kappa, R(t, x)) \varphi d x d t,
\end{aligned}
$$

by the Dominated Convergence Theorem. Furthermore

$$
\begin{aligned}
\Delta & t \sum_{n=0}^{+\infty} \sum_{j=1}^{N}\left[\left(G_{j+1 / 2}^{\kappa}\left(\rho_{j}^{n}, \rho_{j+1}^{n}\right)-G_{j+1 / 2}^{\kappa}\left(\rho_{j}^{n}, \rho_{j}^{n}\right)\right)-\left(G_{j-1 / 2}^{\kappa}\left(\rho_{j-1}^{n}, \rho_{j}^{n}\right)-G_{j+1 / 2}^{\kappa}\left(\rho_{j}^{n}, \rho_{j}^{n}\right)\right)\right] \varphi\left(t^{n}, x_{j}\right) \\
= & \Delta t \sum_{n=0}^{+\infty} \sum_{j=1}^{N}\left(G_{j+1 / 2}^{\kappa}\left(\rho_{j}^{n}, \rho_{j+1}^{n}\right)-G_{j+1 / 2}^{\kappa}\left(\rho_{j}^{n}, \rho_{j}^{n}\right)\right) \varphi\left(t^{n}, x_{j}\right) \\
& -\Delta t \sum_{n=0}^{+\infty} \sum_{j=0}^{N-1}\left(G_{j+1 / 2}^{\kappa}\left(\rho_{j}^{n}, \rho_{j+1}^{n}\right)-G_{j+3 / 2}^{\kappa}\left(\rho_{j+1}^{n}, \rho_{j+1}^{n}\right)\right) \varphi\left(t^{n}, x_{j+1}\right) \\
= & \Delta t \sum_{n=0}^{+\infty} \sum_{j=1}^{N-1}\left[\left(G_{j+1 / 2}^{\kappa}\left(\rho_{j}^{n}, \rho_{j+1}^{n}\right)-G_{j+1 / 2}^{\kappa}\left(\rho_{j}^{n}, \rho_{j}^{n}\right)\right) \varphi\left(t^{n}, x_{j}\right)\right. \\
& \left.-\left(G_{j+1 / 2}^{\kappa}\left(\rho_{j}^{n}, \rho_{j+1}^{n}\right)-G_{j+3 / 2}^{\kappa}\left(\rho_{j+1}^{n}, \rho_{j+1}^{n}\right)\right) \varphi\left(t^{n}, x_{j+1}\right)\right] \\
& +\Delta t \sum_{n=0}^{+\infty}\left(G_{N+1 / 2}^{\kappa}\left(\rho_{N}^{n}, \rho_{b}^{n}\right)-G_{N+1 / 2}^{\kappa}\left(\rho_{N}^{n}, \rho_{N}^{n}\right)\right) \varphi\left(t^{n}, x_{N}\right)-\left(G_{1 / 2}^{\kappa}\left(\rho_{a}^{n}, \rho_{1}^{n}\right)-G_{3 / 2}^{\kappa}\left(\rho_{1}^{n}, \rho_{1}^{n}\right)\right) \varphi\left(t^{n}, x_{1}\right) \\
=: & T_{2}^{i n t}+T_{2}^{b}=: T_{2} .
\end{aligned}
$$


Let us define

$$
\begin{aligned}
T_{20}= & -\Delta t \Delta x \sum_{n \in \mathbb{N}} \sum_{j=1}^{N} G_{j+1 / 2}^{\kappa}\left(\rho_{j}^{n}, \rho_{j}^{n}\right) \frac{\varphi\left(t^{n}, x_{j+1}\right)-\varphi\left(t^{n}, x_{j}\right)}{\Delta x} \\
& -\alpha \Delta t \sum_{n \in \mathbb{N}}\left(\varphi\left(t^{n}, b\right) \eta_{\kappa}^{+}\left(\rho_{b}^{n}\right)+\varphi\left(t^{n}, a\right) \eta_{\kappa}^{+}\left(\rho_{a}^{n}\right)\right) .
\end{aligned}
$$

Being

$$
\begin{aligned}
G_{j+1 / 2}^{\kappa}\left(\rho_{j}^{n}, \rho_{j}^{n}\right)= & F_{j+1 / 2}^{n}\left(\rho_{j}^{n} \wedge \kappa, \rho_{j}^{n} \wedge \kappa\right)-F_{j+1 / 2}^{n}(\kappa, \kappa) \\
= & \frac{1}{2}\left(f\left(t^{n}, x_{j}, \rho_{j}^{n} \wedge \kappa, R_{j}^{n}\right)-f\left(t^{n}, x_{j}, \kappa, R_{j}^{n}\right)\right) \\
& +\frac{1}{2}\left(f\left(t^{n}, x_{j+1}, \rho_{j}^{n} \wedge \kappa, R_{j+1}^{n}\right)-f\left(t^{n}, x_{j+1}, \kappa, R_{j+1}^{n}\right)\right) \\
= & \frac{1}{2} \operatorname{sgn}^{+}\left(\rho_{j}^{n}-\kappa\right)\left(f\left(t^{n}, x_{j}, \rho_{j}^{n}, R_{j}^{n}\right)-f\left(t^{n}, x_{j}, \kappa, R_{j}^{n}\right)\right) \\
& +\frac{1}{2} \operatorname{sgn}^{+}\left(\rho_{j}^{n}-\kappa\right)\left(f\left(t^{n}, x_{j+1}, \rho_{j}^{n}, R_{j+1}^{n}\right)-f\left(t^{n}, x_{j+1}, \kappa, R_{j+1}^{n}\right)\right),
\end{aligned}
$$

it is straightforward to see that

$$
\begin{aligned}
T_{20} \longrightarrow \Delta x \searrow 0^{+} & -\int_{0}^{+\infty} \int_{a}^{b} \operatorname{sgn}^{+}(\rho-\kappa)(f(t, x, \rho, R(t, x))-f(t, x, \kappa, R(t, x))) \partial_{x} \varphi(t, x) d x d t \\
& -\operatorname{Lip}(f) \int_{0}^{+\infty}\left(\rho_{a}(t)-\kappa\right)^{+} \varphi(t, a) d t-\operatorname{Lip}(f) \int_{0}^{+\infty}\left(\rho_{b}(t)-\kappa\right)^{+} \varphi(t, b) d t .
\end{aligned}
$$

We decompose $T_{20}$ as

$$
\begin{aligned}
T_{20}= & -\Delta t \sum_{n=0}^{+\infty} \sum_{j=1}^{N} G_{j+1 / 2}^{\kappa}\left(\rho_{j}^{n}, \rho_{j}^{n}\right)\left(\varphi\left(t^{n}, x_{j+1}\right)-\varphi\left(t^{n}, x_{j}\right)\right) \\
& -\alpha \Delta t \sum_{n=0}^{+\infty}\left(\left(\varphi\left(t^{n}, b\right) \eta_{\kappa}^{+}\left(\rho_{b}^{n}\right)+\varphi\left(t^{n}, a\right) \eta_{\kappa}^{+}\left(\rho_{a}^{n}\right)\right)\right) \\
= & -\Delta t \sum_{n=0}^{+\infty}\left(\sum_{j=1}^{N} G_{j+1 / 2}^{\kappa}\left(\rho_{j}^{n}, \rho_{j}^{n}\right) \varphi\left(t^{n}, x_{j+1}\right)-\sum_{j=0}^{N-1} G_{j+3 / 2}^{\kappa}\left(\rho_{j+1}^{n}, \rho_{j+1}^{n}\right) \varphi\left(t^{n}, x_{j+1}\right)\right) \\
& -\alpha \Delta t \sum_{n=0}^{+\infty}\left(\left(\varphi\left(t^{n}, b\right) \eta_{\kappa}^{+}\left(\rho_{b}^{n}\right)+\varphi\left(t^{n}, a\right) \eta_{\kappa}^{+}\left(\rho_{a}^{n}\right)\right)\right) \\
= & -\Delta t \sum_{n=0}^{+\infty} \sum_{j=1}^{N-1}\left(G_{j+1 / 2}^{\kappa}\left(\rho_{j}^{n}, \rho_{j}^{n}\right)-G_{j+3 / 2}^{\kappa}\left(\rho_{j+1}^{n}, \rho_{j+1}^{n}\right)\right) \varphi\left(t^{n}, x_{j+1}\right) \\
& -\Delta t \sum_{n=0}^{+\infty}\left(G_{N+1 / 2}^{\kappa}\left(\rho_{N}^{n}, \rho_{N}^{n}\right) \varphi\left(t^{n}, x_{N+1}\right)-G_{3 / 2}^{\kappa}\left(\rho_{1}^{n}, \rho_{1}^{n}\right) \varphi\left(t^{n}, x_{1}\right)\right) \\
& -\alpha \Delta t \sum_{n=0}^{+\infty}\left(\left(\varphi\left(t^{n}, b\right) \eta_{\kappa}^{+}\left(\rho_{b}^{n}\right)+\varphi\left(t^{n}, a\right) \eta_{\kappa}^{+}\left(\rho_{a}^{n}\right)\right)\right) \\
=: & T_{20}^{i n t}+T_{20}^{b} .
\end{aligned}
$$


We rewrite

$$
\begin{aligned}
T_{20}^{i n t}= & -\Delta t \sum_{n=0}^{+\infty} \sum_{j=1}^{N-1}\left(G_{j+1 / 2}^{\kappa}\left(\rho_{j}^{n}, \rho_{j}^{n}\right) \mp G_{j+1 / 2}^{\kappa}\left(\rho_{j}^{n}, \rho_{j+1}^{n}\right)-G_{j+3 / 2}^{\kappa}\left(\rho_{j+1}^{n}, \rho_{j+1}^{n}\right)\right) \varphi\left(t^{n}, x_{j+1}\right) \\
= & \Delta t \sum_{n=0}^{+\infty} \sum_{j=1}^{N-1} \varphi\left(t^{n}, x_{j+1}\right)\left(G_{j+1 / 2}^{\kappa}\left(\rho_{j}^{n}, \rho_{j+1}^{n}\right)-G_{j+1 / 2}^{\kappa}\left(\rho_{j}^{n}, \rho_{j}^{n}\right)\right) \\
& -\Delta t \sum_{n=0}^{+\infty} \sum_{j=1}^{N-1} \varphi\left(t^{n}, x_{j+1}\right)\left(G_{j+1 / 2}^{\kappa}\left(\rho_{j}^{n}, \rho_{j+1}^{n}\right)-G_{j+3 / 2}^{\kappa}\left(\rho_{j+1}^{n}, \rho_{j+1}^{n}\right)\right) .
\end{aligned}
$$

Hence

$$
\left|T_{2}^{i n t}-T_{20}^{i n t}\right| \leq \Delta t \sum_{n=0}^{+\infty} \sum_{j=1}^{N-1}\left|\varphi\left(t^{n}, x_{j}\right)-\varphi\left(t^{n}, x_{j+1}\right)\right|\left|G_{j+1 / 2}^{\kappa}\left(\rho_{j}^{n}, \rho_{j+1}^{n}\right)-G_{j+1 / 2}^{\kappa}\left(\rho_{j}^{n}, \rho_{j}^{n}\right)\right| .
$$

Notice that

$$
\begin{aligned}
& \left|G_{j+1 / 2}^{\kappa}\left(\rho_{j}^{n}, \rho_{j+1}^{n}\right)-G_{j+1 / 2}^{\kappa}\left(\rho_{j}^{n}, \rho_{j}^{n}\right)\right|= \\
& =\frac{1}{2}\left|f\left(t^{n}, x_{j+1}, \rho_{j+1}^{n} \wedge \kappa, R_{j+1}^{n}\right)-f\left(t^{n}, x_{j+1}, \rho_{j}^{n} \wedge \kappa, R_{j+1}^{n}\right)+\alpha\left(\rho_{j}^{n} \wedge \kappa-\rho_{j+1}^{n} \wedge \kappa\right)\right| \\
& \leq \frac{L+\alpha}{2}\left|\rho_{j+1}^{n}-\rho_{j}^{n}\right| \\
& \leq \alpha\left|\rho_{j+1}^{n}-\rho_{j}^{n}\right|
\end{aligned}
$$

according to conditions (2.3). Therefore

$$
\begin{aligned}
\left|T_{2}^{i n t}-T_{20}^{i n t}\right| & \leq \alpha \Delta x \Delta t\left\|\varphi_{x}\right\|_{\mathbf{L}^{\infty}} \sum_{n=0}^{+\infty} \sum_{j=1}^{N-1}\left|\rho_{j+1}^{n}-\rho_{j}^{n}\right| \\
& \leq \alpha \Delta x T\left\|\varphi_{x}\right\|_{\mathbf{L}^{\infty}} \max _{0 \leq n \leq T / \Delta t} \operatorname{TV}\left(\rho_{\Delta}\left(t^{n}, \cdot\right)\right)=\mathcal{O}(\Delta x),
\end{aligned}
$$

thanks to the uniform BV bound (2.13). We now compare the terms $T_{2}^{b}$ and $T_{20}^{b}$ :

$$
\begin{aligned}
T_{20}^{b}-T_{2}^{b}= & -\Delta t \sum_{n=0}^{+\infty}\left(G_{N+1 / 2}^{\kappa}\left(\rho_{N}^{n}, \rho_{N}^{n}\right) \varphi\left(t^{n}, x_{N+1}\right)-G_{3 / 2}^{\kappa}\left(\rho_{1}^{n}, \rho_{1}^{n}\right) \varphi\left(t^{n}, x_{1}\right)\right) \\
& -\alpha \Delta t \sum_{n=0}^{+\infty}\left(\varphi\left(t^{n}, b\right) \eta_{\kappa}^{+}\left(\rho_{b}^{n}\right)+\varphi\left(t^{n}, a\right) \eta_{\kappa}^{+}\left(\rho_{a}^{n}\right)\right) \\
& -\Delta t \sum_{n=0}^{+\infty}\left(G_{N+1 / 2}^{\kappa}\left(\rho_{N}^{n}, \rho_{b}^{n}\right)-G_{N+1 / 2}^{\kappa}\left(\rho_{N}^{n}, \rho_{N}^{n}\right)\right) \varphi\left(t^{n}, x_{N}\right) \\
& +\Delta t \sum_{n=0}^{+\infty}\left(G_{1 / 2}^{\kappa}\left(\rho_{a}^{n}, \rho_{1}^{n}\right)-G_{3 / 2}^{\kappa}\left(\rho_{1}^{n}, \rho_{1}^{n}\right)\right) \varphi\left(t^{n}, x_{1}\right) \\
= & \Delta t \sum_{n=0}^{+\infty}\left(G_{1 / 2}^{\kappa}\left(\rho_{a}^{n}, \rho_{1}^{n}\right) \varphi\left(t^{n}, x_{1}\right)-\alpha \eta_{\kappa}^{+}\left(\rho_{a}^{n}\right) \varphi\left(t^{n}, a\right)\right)
\end{aligned}
$$




$$
\begin{aligned}
& -\Delta t \sum_{n=0}^{+\infty}\left(\alpha \eta_{\kappa}^{+}\left(\rho_{b}^{n}\right) \varphi\left(t^{n}, b\right)+G_{N+1 / 2}^{\kappa}\left(\rho_{N}^{n}, \rho_{b}^{n}\right) \varphi\left(t^{n}, x_{N}\right)\right) \\
& -\Delta t \sum_{n=0}^{+\infty}\left(G_{N+1 / 2}^{\kappa}\left(\rho_{N}^{n}, \rho_{N}^{n}\right)\left(\varphi\left(t^{n}, x_{N+1}\right)-\varphi\left(t^{n}, x_{N}\right)\right)\right) \\
= & \Delta t \sum_{n=0}^{+\infty}\left\{\left(G_{1 / 2}^{\kappa}\left(\rho_{a}^{n}, \rho_{1}^{n}\right)-\alpha \eta_{\kappa}^{+}\left(\rho_{a}^{n}\right)\right) \varphi\left(t^{n}, a\right)-\left(\alpha \eta_{\kappa}^{+}\left(\rho_{b}^{n}\right)+G_{N+1 / 2}^{\kappa}\left(\rho_{N}^{n}, \rho_{b}^{n}\right)\right) \varphi\left(t^{n}, b\right)\right\} \\
& +\mathcal{O}(\Delta x),
\end{aligned}
$$

owing to the regularity of $\varphi$. Indeed, we observe that

$$
\begin{aligned}
& \Delta t\left|\sum_{n \in \mathbb{N}}\left(G_{N+1 / 2}^{\kappa}\left(\rho_{N}^{n}, \rho_{N}^{n}\right)\left(\varphi\left(t^{n}, x_{N+1}\right)-\varphi\left(t^{n}, x_{N}\right)\right)\right)\right| \\
& \leq \Delta t \Delta x\left\|\varphi_{x}\right\|_{\mathbf{L}^{\infty}} \sum_{n \in \mathbb{N}}\left|G_{N+1 / 2}^{\kappa}\left(\rho_{N}^{n}, \rho_{N}^{n}\right)\right| \\
& =\Delta t \Delta x\left\|\varphi_{x}\right\|_{\mathbf{L}^{\infty}} \sum_{n \in \mathbb{N}}\left|F_{N+1 / 2}^{n}\left(\rho_{N}^{n} \wedge \kappa, \rho_{N}^{n} \wedge \kappa\right)-F_{N+1 / 2}^{n}(\kappa, \kappa)\right| \\
& \leq \frac{\Delta t \Delta x\left\|\varphi_{x}\right\|_{\mathbf{L}^{\infty}}}{2} \sum_{n \in \mathbb{N}}\left(\left|f\left(t^{n}, x_{N}, \rho_{N}^{n} \wedge \kappa, R_{N}^{n}\right)-f\left(t^{n}, x_{N}, \kappa, R_{N}^{n}\right)\right|\right) \\
& \quad+\frac{\Delta t \Delta x\left\|_{x}\right\|_{\mathbf{L}^{\infty}}}{2} \sum_{n \in \mathbb{N}}\left(\left|f\left(t^{n}, x_{N+1}, \rho_{N}^{n} \wedge \kappa, R_{N+1}^{n}\right)-f\left(t^{n}, x_{N+1}, \kappa, R_{N+1}^{n}\right)\right|\right) \\
& \leq L \Delta t \Delta x\left\|\varphi_{x}\right\|_{\mathbf{L}^{\infty}} \sum_{n \in \mathbb{N}}\left(\rho_{N}^{n}-\kappa\right)^{+} \\
& \leq L T\left\|\varphi_{x}\right\|_{\mathbf{L}^{\infty}} e^{\mathcal{L} T}\left\|\rho_{0}\right\|_{\mathbf{L}^{\infty}(I)} \Delta x=\mathcal{O}(\Delta x)
\end{aligned}
$$

thanks to the $\mathbf{L}^{\infty}$-bound (2.9). Moreover, since

$$
\begin{aligned}
G_{j+1 / 2}^{\kappa}(u, v) & =F_{j+1 / 2}^{n}(u \wedge \kappa, v \wedge \kappa)-F_{j+1 / 2}^{n}(\kappa, \kappa) \\
& \geq F_{j+1 / 2}^{n}(\kappa, v \wedge \kappa)-F_{j+1 / 2}^{n}(\kappa, \kappa) \\
& =\frac{1}{2}\left(f\left(t^{n}, x_{j+1}, v \wedge \kappa, R_{j+1}^{n}\right)-f\left(t^{n}, x_{j+1}, \kappa, R_{j+1}^{n}\right)-\alpha(v \wedge \kappa-\kappa)\right) \\
& \geq-\frac{1}{2}\left(L|v \wedge \kappa-\kappa|+\alpha(v-\kappa)^{+}\right) \\
& =-\frac{1}{2}\left(L(v-\kappa)^{+}+\alpha(v-\kappa)^{+}\right) \\
& \geq-\alpha(v-\kappa)^{+}
\end{aligned}
$$

and

$$
\begin{aligned}
G_{j+1 / 2}^{\kappa}(u, v) & =F_{j+1 / 2}^{n}(u \wedge \kappa, v \wedge \kappa)-F_{j+1 / 2}^{n}(\kappa, \kappa) \\
& \leq F_{j+1 / 2}^{n}(u \wedge \kappa, \kappa)-F_{j+1 / 2}^{n}(\kappa, \kappa) \\
& =\frac{1}{2}\left(f\left(t^{n}, x_{j}, u \wedge \kappa, R_{j}^{n}\right)-f\left(t^{n}, x_{j}, \kappa, R_{j}^{n}\right)+\alpha(u \wedge \kappa-\kappa)\right) \\
& \leq \frac{1}{2}\left(L|u \wedge \kappa-\kappa|+\alpha(u-\kappa)^{+}\right)
\end{aligned}
$$




$$
\begin{aligned}
& =\frac{1}{2}\left(L(u-\kappa)^{+}+\alpha(u-\kappa)^{+}\right) \\
& \leq \alpha(u-\kappa)^{+}
\end{aligned}
$$

we conclude that

$$
T_{20}^{b}-T_{2}^{b} \leq \mathcal{O}(\Delta x)
$$

Therefore we get

$$
\begin{aligned}
0 & \geq T_{1}+T_{3}+T_{2} \\
& =T_{1}+T_{3}+T_{2}^{i n t}+T_{2}^{b} \\
& \geq T_{1}+T_{3}+T_{2}^{i n t}+T_{20}^{b}-\mathcal{O}(\Delta x) \\
& =T_{1}+T_{3}+T_{20}-\mathcal{O}(\Delta x),
\end{aligned}
$$

thus concluding the proof.

\section{Stability}

Proposition 2 Under hypotheses (1.2), let $\rho, \sigma \in \mathbf{C}^{\mathbf{0}}\left(\mathbb{R}^{+} ; \mathbf{L}^{\mathbf{1}}\left(I ; \mathbb{R}^{+}\right)\right) \cap B V([0, T] \times I ; \mathbb{R}), T>0$, be two weak entropy solutions to (1.1), with initial data $\rho_{0}, \sigma_{0} \in \mathbf{L}^{\infty}\left(I, \mathbb{R}^{+}\right)$and boundary data $\rho_{a}, \rho_{b}, \sigma_{a}, \sigma_{b} \in \mathbf{L}^{\infty}\left(\mathbb{R}^{+} ; \mathbb{R}^{+}\right)$respectively. Then the following estimate holds:

$$
\|\rho(T, \cdot)-\sigma(T, \cdot)\|_{\mathbf{L}^{1}(I)} \leq e^{\mathcal{S} T}\left[\left\|\rho_{0}-\sigma_{0}\right\|_{\mathbf{L}^{1}(I)}+\left(L+\mathcal{S}^{\prime}\right)\left(\left\|\rho_{a}-\sigma_{a}\right\|_{\mathbf{L}^{1}([0, T])}+\left\|\rho_{b}-\sigma_{b}\right\|_{\mathbf{L}^{1}([0, T])}\right)\right],
$$

where the constants $\mathcal{S}, \mathcal{S}^{\prime}$ are defined by (3.6), (3.7) and (3.10), and $L$ is as in (2.28).

Proof. Let $\rho, \sigma$ be two weak entropy solutions to (1.1), with fluxes $f(t, x, \rho, R(t, x))$ and $f(t, x, \sigma, S(t, x))$ respectively, where

$$
R(t, x):=\int_{\mathbb{R}} \rho(t, y) \eta(x-y) d y \quad \text { and } \quad S(t, x):=\int_{\mathbb{R}} \sigma(t, y) \eta(x-y) d y .
$$

In particular from Definition 2, they satisfy

$$
\begin{aligned}
\partial_{t}|\rho-\kappa| & +\frac{d}{d x}[\operatorname{sgn}(\rho-\kappa)(f(t, x, \rho, R(t, x))-f(t, x, \kappa, R(t, x)))] \\
& +\operatorname{sgn}(\rho-\kappa) \frac{d}{d x} f(t, x, \kappa, R(t, x)) \leq 0, \\
\partial_{t}|\sigma-\kappa|+ & \frac{d}{d x}[\operatorname{sgn}(\sigma-\kappa)(f(t, x, \sigma, S(t, x))-f(t, x, \kappa, S(t, x)))] \\
& +\operatorname{sgn}(\sigma-\kappa) \frac{d}{d x} f(t, x, \kappa, S(t, x)) \leq 0,
\end{aligned}
$$

in distributional sense on $\mathbb{R}^{+} \times I$. Rearranging (3.2) we get

$0 \geq \partial_{t}|\rho-\kappa|+\operatorname{sgn}(\rho-\kappa) \frac{d}{d x}(f(t, x, \kappa, R(t, x)) \pm f(t, x, \kappa, S(t, x)))$ 


$$
\begin{aligned}
& +\frac{d}{d x}[\operatorname{sgn}(\rho-\kappa)(f(t, x, \rho, R(t, x))-f(t, x, \kappa, R(t, x)) \pm f(t, x, \rho, S(t, x)) \pm f(t, x, \kappa, S(t, x)))] \\
= & \partial_{t}|\rho-\kappa|+\frac{d}{d x}[\operatorname{sgn}(\rho-\kappa)(f(t, x, \rho, S(t, x))-f(t, x, \kappa, S(t, x)))]+\operatorname{sgn}(\rho-\kappa) \frac{d}{d x} f(t, x, \kappa, S(t, x)) \\
& +\frac{d}{d x}[\operatorname{sgn}(\rho-\kappa)((f(t, x, \rho, R(t, x))-f(t, x, \rho, S(t, x)))-(f(t, x, \kappa, R(t, x))-f(t, x, \kappa, S(t, x))))] \\
& +\operatorname{sgn}(\rho-\kappa) \frac{d}{d x}[f(t, x, \kappa, R(t, x))-f(t, x, \kappa, S(t, x))],
\end{aligned}
$$

thus

$$
\begin{aligned}
& \partial_{t}|\rho-\kappa|+\frac{d}{d x}[\operatorname{sgn}(\rho-\kappa)(f(t, x, \rho, S(t, x))-f(t, x, \kappa, S(t, x)))]+\operatorname{sgn}(\rho-\kappa) \frac{d}{d x} f(t, x, \kappa, S(t, x)) \\
& \leq \frac{d}{d x}\{\operatorname{sgn}(\rho-\kappa)[(f(t, x, \rho, S(t, x))-f(t, x, \rho, R(t, x)))-(f(t, x, \kappa, S(t, x))-f(t, x, \kappa, R(t, x)))]\} \\
& \quad+\operatorname{sgn}(\rho-\kappa) \frac{d}{d x}[f(t, x, \kappa, S(t, x))-f(t, x, \kappa, R(t, x))] .
\end{aligned}
$$

Notice that from (1.5) we can bound

$$
\begin{gathered}
|R(t, x)-S(t, x)| \leq\|\eta\|_{\mathbf{L}^{\infty}} \int_{a}^{b}|\rho(t, x)-\sigma(t, x)| d x+\left|\rho_{a}(t)-\sigma_{a}(t)\right|+\left|\rho_{b}(t)-\sigma_{b}(t)\right|, \\
\left|\partial_{x} R(t, x)-\partial_{x} S(t, x)\right| \leq\left\|\partial_{x} \eta\right\|_{\mathbf{L}^{\infty}} \int_{a}^{b}|\rho(t, x)-\sigma(t, x)| d x+\|\eta\|_{\mathbf{L}^{\infty}}\left(\left|\rho_{a}(t)-\sigma_{a}(t)\right|+\left|\rho_{b}(t)-\sigma_{b}(t)\right|\right) .
\end{gathered}
$$

Therefore we recover the following estimate:

$$
\begin{aligned}
& \left|\frac{d}{d x}(f(t, x, \kappa, S(t, x))-f(t, x, \kappa, R(t, x)))\right| \leq \\
\leq & \left|\partial_{x} f(t, x, \kappa, S(t, x))-\partial_{x} f(t, x, \kappa, R(t, x))\right| \\
& +\left|\partial_{R} f(t, x, \kappa, S(t, x)) \partial_{x} S(t, x)-\partial_{R} f(t, x, \kappa, R(t, x)) \partial_{x} R(t, x) \pm \partial_{R} f(t, x, \kappa, S(t, x)) \partial_{x} R(t, x)\right| \\
\leq & \left|\partial_{x R}^{2} f\left(t, x, \kappa, \tilde{R}_{1}(t, x)\right)\right||S(t, x)-R(t, x)| \\
& +\left|\partial_{R} f(t, x, \kappa, S(t, x))\right|\left|\partial_{x} S(t, x)-\partial_{x} R(t, x)\right| \\
& +\left|\partial_{R} f(t, x, \kappa, S(t, x))-\partial_{R} f(t, x, \kappa, R(t, x))\right|\left|\partial_{x} R(t, x)\right| \\
\leq & \left(\left|\partial_{x R}^{2} f\left(t, x, \kappa, \tilde{R}_{1}(t, x)\right)\right|+\left|\partial_{R R}^{2} f\left(t, x, \kappa, \tilde{R}_{2}(t, x)\right)\right|\left|\partial_{x} R(t, x)\right|\right)|S(t, x)-R(t, x)| \\
& +\left|\partial_{R} f(t, x, \kappa, S(t, x))\right|\left|\partial_{x} S(t, x)-\partial_{x} R(t, x)\right| \\
\leq & C|\kappa|\left(1+\left|\partial_{x} R(t, x)\right|\right)|S(t, x)-R(t, x)|+C|\kappa|\left|\partial_{x} S(t, x)-\partial_{x} R(t, x)\right| \\
= & C|\kappa|\left(1+\left|\int_{\mathbb{R}} \partial_{x} \eta(x-y) \rho(t, y) d y\right|\right)|S(t, x)-R(t, x)|+C|\kappa|\left|\partial_{x} S(t, x)-\partial_{x} R(t, x)\right| \\
\leq & C|\kappa|\left(1+\|\rho(t, \cdot)\|_{\mathbf{L}^{\infty}}\left\|\partial_{x} \eta\right\|_{\mathbf{L}^{1}}\right)|S(t, x)-R(t, x)|+C\left|\kappa \| \partial_{x} S(t, x)-\partial_{x} R(t, x)\right| \\
\leq & C|\kappa|\left(1+\|\rho(t, \cdot)\|_{\mathbf{L}^{\infty}}\left\|\partial_{x} \eta\right\|_{\mathbf{L}^{1}}\right)\left[\|\eta\|_{\mathbf{L}^{\infty}} \int_{a}^{b}|\rho(t, x)-\sigma(t, x)| d x+\left|\rho_{a}(t)-\sigma_{a}(t)\right|+\left|\rho_{b}(t)-\sigma_{b}(t)\right|\right] \\
& +C|\kappa|\left[\left\|\partial_{x} \eta\right\|_{\mathbf{L}^{\infty}} \int_{a}^{b}|\rho(t, x)-\sigma(t, x)| d x+\|\eta\|_{\mathbf{L}^{\infty}}\left(\left|\rho_{a}(t)-\sigma_{a}(t)\right|+\left|\rho_{b}(t)-\sigma_{b}(t)\right|\right)\right]
\end{aligned}
$$




$$
\begin{aligned}
\leq & C|\kappa|\left[\|\eta\|_{\mathbf{L}^{\infty}}\left(1+\|\rho(t, \cdot)\|_{\mathbf{L}^{\infty}}\left\|\partial_{x} \eta\right\|_{\mathbf{L}^{1}}\right)+\left\|\partial_{x} \eta\right\|_{\mathbf{L}^{\infty}}\right] \int_{a}^{b}|\rho(t, x)-\sigma(t, x)| d x \\
& +C|\kappa|\left(1+\|\rho(t, \cdot)\|_{\mathbf{L}^{\infty}}\left\|\partial_{x} \eta\right\|_{\mathbf{L}^{1}}+\|\eta\|_{\mathbf{L}^{\infty}}\right)\left(\left|\rho_{a}(t)-\sigma_{a}(t)\right|+\left|\rho_{b}(t)-\sigma_{b}(t)\right|\right) \\
\leq & \mathcal{S}_{1} \int_{a}^{b}|\rho(t, y)-\sigma(t, y)| d y+\mathcal{S}_{1}^{\prime}\left(\left|\rho_{a}(t)-\sigma_{a}(t)\right|+\left|\rho_{b}(t)-\sigma_{b}(t)\right|\right),
\end{aligned}
$$

where

$$
\begin{aligned}
& \mathcal{S}_{1}=C \sup _{t \in[0, T]}\left\{\max \left\{\|\rho(t, \cdot)\|_{\mathbf{L}^{\infty}},\|\sigma(t, \cdot)\|_{\mathbf{L}^{\infty}}\right\}\left[\|\eta\|_{\mathbf{L}^{\infty}}\left(1+\|\rho(t, \cdot)\|_{\mathbf{L}^{\infty}}\left\|\partial_{x} \eta\right\|_{\mathbf{L}^{1}}\right)+\left\|\partial_{x} \eta\right\|_{\mathbf{L}^{\infty}}\right]\right\} \\
& \mathcal{S}_{1}^{\prime}=C \sup _{t \in[0, T]}\left\{\max \left\{\|\rho(t, \cdot)\|_{\mathbf{L}^{\infty}},\|\sigma(t, \cdot)\|_{\mathbf{L}^{\infty}}\right\}\left(1+\|\rho(t, \cdot)\|_{\mathbf{L}^{\infty}}\left\|\partial_{x} \eta\right\|_{\mathbf{L}^{1}}+\|\eta\|_{\mathbf{L}^{\infty}}\right)\right\},
\end{aligned}
$$

which are bounded by assumption. Moreover

$$
\begin{aligned}
& \left|\frac{d}{d x}\{\operatorname{sgn}(\rho-\kappa)[(f(t, x, \rho, S(t, x))-f(t, x, \rho, R(t, x)))-(f(t, x, \kappa, S(t, x))-f(t, x, \kappa, R(t, x)))]\}\right| \\
= & \mid\left\{\partial_{x} f(t, x, \rho, S(t, x))-\partial_{x} f(t, x, \rho, R(t, x))+\partial_{x} f(t, x, \kappa, R(t, x))-\partial_{x} f(t, x, \kappa, S(t, x))\right. \\
& +\partial_{R} f(t, x, \rho, S(t, x))\left(\partial_{x} S(t, x)-\partial_{x} R(t, x)\right)+\partial_{x} R(t, x)\left(\partial_{x} f(t, x, \rho, S(t, x))-\partial_{x} f(t, x, \rho, R(t, x))\right) \\
& \left.+\partial_{R} f(t, x, \kappa, S(t, x))\left(\partial_{x} R(t, x)-\partial_{x} S(t, x)\right)+\partial_{x} R(t, x)\left(\partial_{x} f(t, x, \kappa, R(t, x))-\partial_{x} f(t, x, \kappa, S(t, x))\right)\right\} \mid \\
= & \mid \partial_{x R}^{2} f\left(t, x, \rho, \tilde{R}_{1}\right)(S(t, x)-R(t, x))+\partial_{x R}^{2} f\left(t, x, \kappa, \tilde{R}_{2}\right)(R(t, x)-S(t, x)) \\
& +\partial_{R} f(t, x, \rho, S(t, x))\left(\partial_{x} S(t, x)-\partial_{x} R(t, x)\right)+\partial_{x} R(t, x) \partial_{x R}^{2} f\left(t, x, \rho, \tilde{R}_{3}\right)(S(t, x)-R(t, x)) \\
& +\partial_{R} f(t, x, \kappa, S(t, x))\left(\partial_{x} R(t, x)-\partial_{x} S(t, x)\right)+\partial_{x} R(t, x) \partial_{x R}^{2} f\left(t, x, \kappa, \tilde{R}_{4}\right)(R(t, x)-S(t, x)) \mid \\
\leq & C\left(\|\rho(t, \cdot)\|_{\mathbf{L} \infty}+|\kappa|\right)\left(1+\|\rho(t, \cdot)\|_{\mathbf{L} \infty}\left\|\partial_{x} \eta\right\|_{\mathbf{L}^{1}}\right)|R(t, x)-S(t, x)| \\
& +C\left(\|\rho(t, \cdot)\|_{\mathbf{L} \infty}+|\kappa|\right)\left|\partial_{x} R(t, x)-\partial_{x} S(t, x)\right| \\
= & \mathcal{S}_{2} \int_{a}^{b}|\rho(t, y)-\sigma(t, y)| d y+\mathcal{S}_{2}^{\prime}\left(\left|\rho_{a}(t)-\sigma_{a}(t)\right|+\left|\rho_{b}(t)-\sigma_{b}(t)\right|\right),
\end{aligned}
$$

being $\mathcal{S}_{2}=2 \mathcal{S}_{1}$ and $\mathcal{S}_{2}^{\prime}=2 \mathcal{S}_{1}^{\prime}$.

Inserting estimates (3.5) and (3.8) in (3.4) we get

$$
\begin{aligned}
& \partial_{t}|\rho-\kappa|+\frac{d}{d x}[\operatorname{sgn}(\rho-\kappa)(f(t, x, \rho, S(t, x))-f(t, x, \kappa, S(t, x)))]+\operatorname{sgn}(\rho-\kappa) \frac{d}{d x} f(t, x, \kappa, S(t, x)) \\
& \leq \mathcal{S} \int_{a}^{b}|\rho(t, y)-\sigma(t, y)| d y+\mathcal{S}^{\prime}\left(\left|\rho_{a}(t)-\sigma_{a}(t)\right|+\left|\rho_{b}(t)-\sigma_{b}(t)\right|\right),
\end{aligned}
$$

with

$$
\mathcal{S}=\mathcal{S}_{1}+\mathcal{S}_{2}=3 \mathcal{S}_{1} \quad \text { and } \quad \mathcal{S}^{\prime}=\mathcal{S}_{1}^{\prime}+\mathcal{S}_{2}^{\prime}=3 \mathcal{S}_{1}^{\prime}
$$

Following [6, Theorem 2] and [25, Theorem 15.1.5], we apply the standard Kružhkov doubling of variable technique $\left[20\right.$, Section 3] to (3.9) and (3.3), with a test function $\varphi \in \mathcal{C}_{c}^{1}\left(\mathbb{R} \times I ; \mathbb{R}^{+}\right)$. We obtain the following Kato inequality

$$
\int_{a}^{b}\left|\rho_{0}(x)-\sigma_{0}(x)\right| \varphi(0, x) d x
$$




$$
\begin{aligned}
& +\int_{0}^{+\infty} \int_{a}^{b}\left(|\rho(t, x)-\sigma(t, x)| \partial_{t} \varphi+\operatorname{sgn}(\rho-\sigma)[f(t, x, \rho, S(t, x))-f(t, x, \sigma, S(t, x))] \partial_{x} \varphi\right) d x d t \\
& +\mathcal{S}\|\varphi\|_{\mathbf{L}^{\infty}} \int_{0}^{+\infty}\left(\int_{a}^{b}|\rho(t, x)-\sigma(t, x)| d x\right) d t+\mathcal{S}^{\prime}\|\varphi\|_{\mathbf{L}^{\infty}} \int_{0}^{+\infty}\left(\left|\rho_{a}(t)-\sigma_{a}(t)\right|+\left|\rho_{b}(t)-\sigma_{b}(t)\right|\right) d t \\
& \geq 0 .
\end{aligned}
$$

We now consider in (3.11) a test function of the form $\varphi(t, x)=\psi(t) \theta_{\delta}(x)$, where $\theta_{\delta} \in \mathcal{C}^{1}([a, b])$ be such that

$$
\begin{aligned}
& \theta_{\delta}(a)=0, \theta_{\delta}(b)=0 \\
& \left\|\theta_{\delta}^{\prime}\right\|_{\infty} \leq K / \delta \\
& \theta_{\delta} \equiv 1 \text { on }[a+\delta, b-\delta] \\
& 0 \leq \theta_{\delta}(x) \leq 1 \text { for all } x \in[a, b]
\end{aligned}
$$

where $C$ does not depend on $\delta$, and $\psi \in \mathcal{C}_{c}^{1}([0, T[)$. In this case, (3.11) becomes

$$
\begin{aligned}
& \int_{a}^{b}\left|\rho_{0}(x)-\sigma_{0}(x)\right| \psi(0) \theta_{\delta}(x) d x \\
& +\int_{0}^{+\infty} \int_{a}^{b}\left(|\rho(t, x)-\sigma(t, x)| \theta_{\delta}(x) \psi^{\prime}(t)\right. \\
& \left.\quad+\psi(t) \theta_{\delta}^{\prime}(x) \operatorname{sgn}(\rho-\sigma)[f(t, x, \rho, S(t, x))-f(t, x, \sigma, S(t, x))]\right) d x d t \\
& \quad+\mathcal{S}\|\varphi\|_{\mathbf{L}^{\infty}} \int_{0}^{T}\left(\int_{a}^{b}|\rho(t, x)-\sigma(t, x)| d x\right) d t+\mathcal{S}^{\prime}\|\varphi\|_{\mathbf{L}^{\infty}} \int_{0}^{T}\left(\left|\rho_{a}(t)-\sigma_{a}(t)\right|+\left|\rho_{b}(t)-\sigma_{b}(t)\right|\right) d t \\
& \geq 0 .
\end{aligned}
$$

Integrating by parts in (3.12) and letting $\delta \searrow 0$ we obtain

$$
\begin{aligned}
& \int_{a}^{b}\left|\rho_{0}(x)-\sigma_{0}(x)\right| \psi(0) d x \\
& +\int_{0}^{+\infty} \int_{a}^{b}|\rho(t, x)-\sigma(t, x)| \psi^{\prime}(t) d x d t+\mathcal{S}\|\varphi\|_{\mathbf{L}^{\infty}} \int_{0}^{T}\left(\int_{a}^{b}|\rho(t, x)-\sigma(t, x)| d x\right) d t \\
& +\mathcal{S}^{\prime}\|\varphi\|_{\mathbf{L}^{\infty}} \int_{0}^{T}\left(\left|\rho_{a}(t)-\sigma_{a}(t)\right|+\left|\rho_{b}(t)-\sigma_{b}(t)\right|\right) d t \\
& +\int_{0}^{+\infty} \psi(t)\left\{\operatorname{sgn}\left(\rho\left(t, a^{+}\right)-\sigma\left(t, a^{+}\right)\right)\left[f\left(t, a, \rho\left(t, a^{+}\right), S(t, a)\right)-f\left(t, a, \sigma\left(t, a^{+}\right), S(t, a)\right)\right]\right. \\
& \left.\quad-\operatorname{sgn}\left(\rho\left(t, b^{-}\right)-\sigma\left(t, b^{-}\right)\right)\left[f\left(t, b, \rho\left(t, b^{-}\right), S(t, b)\right)-f\left(t, b, \sigma\left(t, b^{-}\right), S(t, b)\right)\right]\right\} d t \geq 0 .
\end{aligned}
$$

From the weak boundary conditions (1.7) and (1.8), we earn

$$
\begin{aligned}
& \operatorname{sgn}\left(\rho\left(t, a^{+}\right)-\sigma\left(t, a^{+}\right)\right)\left[f\left(t, a, \rho\left(t, a^{+}\right), S(t, a)\right)-f\left(t, a, \sigma\left(t, a^{+}\right), S(t, a)\right)\right] \\
= & \frac{1}{2} \operatorname{sgn}\left(\rho\left(t, a^{+}\right)-\sigma\left(t, a^{+}\right)\right)\left[f\left(t, a, \rho\left(t, a^{+}\right), S(t, a)\right)-f\left(t, a, \sigma\left(t, a^{+}\right), S(t, a)\right)\right]
\end{aligned}
$$




$$
\begin{aligned}
& +\frac{1}{2} \operatorname{sgn}\left(\rho\left(t, a^{+}\right)-\sigma\left(t, a^{+}\right)\right)\left[f\left(t, a, \rho\left(t, a^{+}\right), S(t, a)\right)-f\left(t, a, \sigma\left(t, a^{+}\right), S(t, a)\right)\right] \\
\leq & \frac{1}{2} \operatorname{sgn}\left(\rho_{a}(t)-\sigma\left(t, a^{+}\right)\right)\left[f\left(t, a, \rho\left(t, a^{+}\right), S(t, a)\right)-f\left(t, a, \sigma\left(t, a^{+}\right), S(t, a)\right)\right] \\
& +\frac{1}{2} \operatorname{sgn}\left(\rho\left(t, a^{+}\right)-\sigma_{a}(t)\right)\left[f\left(t, a, \rho\left(t, a^{+}\right), S(t, a)\right)-f\left(t, a, \sigma\left(t, a^{+}\right), S(t, a)\right)\right] \\
= & \frac{1}{2}\left(\operatorname{sgn}\left(\rho_{a}(t)-\sigma\left(t, a^{+}\right)\right)+\operatorname{sgn}\left(\rho\left(t, a^{+}\right)-\sigma_{a}(t)\right)\right)\left[f\left(t, a, \rho\left(t, a^{+}\right), S(t, a)\right)-f\left(t, a, \sigma\left(t, a^{+}\right), S(t, a)\right)\right] \\
\leq & \sup _{s, r \in \mathcal{I}\left(\rho_{a}(t), \sigma_{a}(t)\right)}|f(t, a, s, S(t, a))-f(t, a, r, S(t, a))| \\
\leq & \sup _{s, r \in \mathcal{I}\left(\rho_{a}(t), \sigma_{a}(t)\right)} L|s-r| \\
\leq & L\left|\rho_{a}(t)-\sigma_{a}(t)\right|,
\end{aligned}
$$

and

$$
\begin{aligned}
& \operatorname{sgn}\left(\rho\left(t, b^{-}\right)-\sigma\left(t, b^{-}\right)\right)\left[f\left(t, b, \rho\left(t, b^{-}\right), S(t, b)\right)-f\left(t, b, \sigma\left(t, b^{-}\right), S(t, b)\right)\right] \\
= & \frac{1}{2} \operatorname{sgn}\left(\rho\left(t, b^{-}\right)-\sigma\left(t, b^{-}\right)\right)\left[f\left(t, b, \rho\left(t, b^{-}\right), S(t, b)\right)-f\left(t, b, \sigma\left(t, b^{-}\right), S(t, b)\right)\right] \\
& +\frac{1}{2} \operatorname{sgn}\left(\rho\left(t, b^{-}\right)-\sigma\left(t, b^{-}\right)\right)\left[f\left(t, b, \rho\left(t, b^{-}\right), S(t, b)\right)-f\left(t, b, \sigma\left(t, b^{-}\right), S(t, b)\right)\right] \\
\geq & \frac{1}{2} \operatorname{sgn}\left(\rho_{b}(t)-\sigma\left(t, b^{-}\right)\right)\left[f\left(t, b, \rho\left(t, b^{-}\right), S(t, b)\right)-f\left(t, b, \sigma\left(t, b^{-}\right), S(t, b)\right)\right] \\
& +\frac{1}{2} \operatorname{sgn}\left(\rho\left(t, b^{-}\right)-\sigma_{b}(t)\right)\left[f\left(t, b, \rho\left(t, b^{-}\right), S(t, b)\right)-f\left(t, b, \sigma\left(t, b^{-}\right), S(t, b)\right)\right] \\
= & \frac{1}{2}\left(\operatorname{sgn}\left(\rho_{b}(t)-\sigma\left(t, b^{-}\right)\right)+\operatorname{sgn}\left(\rho\left(t, b^{-}\right)-\sigma_{b}(t)\right)\right)\left[f\left(t, b, \rho\left(t, b^{-}\right), S(t, b)\right)-f\left(t, b, \sigma\left(t, b^{-}\right), S(t, b)\right)\right] \\
\geq & -\sup _{s, r \in \mathcal{I}\left(\rho_{b}(t), \sigma_{b}(t)\right)}|f(t, b, s, S(t, b))-f(t, b, r, S(t, b))| \\
\geq & -\sup _{s, r \in \mathcal{I}\left(\rho_{b}(t), \sigma_{b}(t)\right)} L|s-r| \\
\geq & -L\left|\rho_{b}(t)-\sigma_{b}(t)\right| .
\end{aligned}
$$

Collecting (3.14) and (3.15) we conclude that

$$
\begin{aligned}
& \int_{0}^{+\infty} \psi(t)\left\{\operatorname{sgn}\left(\rho\left(t, a^{+}\right)-\sigma\left(t, a^{+}\right)\right)\left[f\left(t, a, \rho\left(t, a^{+}\right), S(t, a)\right)-f\left(t, a, \sigma\left(t, a^{+}\right), S(t, a)\right)\right]\right. \\
& \left.\quad-\operatorname{sgn}\left(\rho\left(t, b^{-}\right)-\sigma\left(t, b^{-}\right)\right)\left[f\left(t, b, \rho\left(t, b^{-}\right), S(t, b)\right)-f\left(t, b, \sigma\left(t, b^{-}\right), S(t, b)\right)\right]\right\} d t \\
& \leq L \int_{0}^{+\infty} \psi(t)\left(\left|\rho_{a}(t)-\sigma_{a}(t)\right|+\left|\rho_{b}(t)-\sigma_{b}(t)\right|\right) d t .
\end{aligned}
$$

Thus (3.13) becomes

$$
\begin{aligned}
& \int_{a}^{b}\left|\rho_{0}(x)-\sigma_{0}(x)\right| \psi(0) d x \\
& +\int_{0}^{+\infty} \int_{a}^{b}|\rho(t, x)-\sigma(t, x)| \psi^{\prime}(t) d x d t+\mathcal{S}\|\varphi\|_{\mathbf{L}^{\infty}} \int_{0}^{T}\left(\int_{a}^{b}|\rho(t, x)-\sigma(t, x)| d x\right) d t
\end{aligned}
$$




$$
\begin{aligned}
& +\mathcal{S}^{\prime}\|\varphi\|_{\mathbf{L}^{\infty}} \int_{0}^{T}\left(\left|\rho_{a}(t)-\sigma_{a}(t)\right|+\left|\rho_{b}(t)-\sigma_{b}(t)\right|\right) d t \\
& +L \int_{0}^{+\infty} \psi(t)\left(\left|\rho_{a}(t)-\sigma_{a}(t)\right|+\left|\rho_{b}(t)-\sigma_{b}(t)\right|\right) d t \geq 0 .
\end{aligned}
$$

We choose the test function $\psi=\psi_{\epsilon}$ as

$$
\psi_{\epsilon}(t)= \begin{cases}1 & \text { if } t \in[0, T-\epsilon[ \\ \psi_{\epsilon}(t) \in[0,1] & \text { for all } t \in[0, T] \\ \left|\psi_{\epsilon}^{\prime}(t)\right| \leq K / \epsilon & \text { for all } t \in[0, T]\end{cases}
$$

As $\epsilon \searrow 0$, we get

$$
\begin{aligned}
\|\rho(T, \cdot)-\sigma(T, \cdot)\|_{\mathbf{L}^{\mathbf{1}}(I)} \leq\left\|\rho_{0}-\sigma_{0}\right\|_{\mathbf{L}^{\mathbf{1}}(I)}+L\left(\left\|\rho_{b}-\sigma_{b}\right\|_{\mathbf{L}^{\mathbf{1}}([0, T])}+\left\|\rho_{a}-\sigma_{a}\right\|_{\mathbf{L}^{\mathbf{1}}([0, T])}\right) \\
+\mathcal{S} \int_{0}^{T}\|\rho(t, \cdot)-\sigma(t, \cdot)\|_{\mathbf{L}^{\mathbf{1}}(I)} d t+\mathcal{S}^{\prime} \int_{0}^{T}\left(\left|\rho_{a}(t)-\sigma_{a}(t)\right|+\left|\rho_{b}(t)-\sigma_{b}(t)\right|\right) d t,
\end{aligned}
$$

and Gronwall's lemma allows us to recover (3.1).

\section{References}

[1] A. Aggarwal, R. M. Colombo, and P. Goatin. Nonlocal systems of conservation laws in several space dimensions. SIAM J. Numer. Anal., 53(2):963-983, 2015.

[2] D. Amadori, S.-Y. Ha, and J. Park. On the global well-posedness of the bv weak solutions to the Kuramoto-Sakaguchi equation. Submitted.

[3] D. Amadori and W. Shen. An integro-differential conservation law arising in a model of granular flow. J. Hyperbolic Differ. Equ., 9(1):105-131, 2012.

[4] P. Amorim. On a nonlocal hyperbolic conservation law arising from a gradient constraint problem. Bull. Braz. Math. Soc. (N.S.), 43(4):599-614, 2012.

[5] P. Amorim, R. Colombo, and A. Teixeira. On the numerical integration of scalar nonlocal conservation laws. ESAIM M2AN, 49(1):19-37, 2015.

[6] C. Bardos, A. Y. le Roux, and J.-C. Nédélec. First order quasilinear equations with boundary conditions. Comm. Partial Differential Equations, 4(9):1017-1034, 1979.

[7] F. Betancourt, R. Bürger, K. H. Karlsen, and E. M. Tory. On nonlocal conservation laws modelling sedimentation. Nonlinearity, 24(3):855-885, 2011.

[8] S. Blandin and P. Goatin. Well-posedness of a conservation law with non-local flux arising in traffic flow modeling. Numer. Math., 132(2):217-241, 2016.

[9] F. Bouchut and B. Perthame. Kružkov's estimates for scalar conservation laws revisited. Trans. Amer. Math. Soc., 350(7):2847-2870, 1998.

[10] J. A. Carrillo, S. Martin, and M.-T. Wolfram. An improved version of the Hughes model for pedestrian flow. Math. Models Methods Appl. Sci., 26(4):671-697, 2016.

[11] R. M. Colombo, M. Garavello, and M. Lécureux-Mercier. A class of nonlocal models for pedestrian traffic. Mathematical Models and Methods in Applied Sciences, 22(04):1150023, 2012.

[12] R. M. Colombo, M. Herty, and M. Mercier. Control of the continuity equation with a non local flow. ESAIM Control Optim. Calc. Var., 17(2):353-379, 2011. 
[13] R. M. Colombo and M. Lécureux-Mercier. Nonlocal crowd dynamics models for several populations. Acta Math. Sci. Ser. B Engl. Ed., 32(1):177-196, 2012.

[14] G. Crippa and M. Lécureux-Mercier. Existence and uniqueness of measure solutions for a system of continuity equations with non-local flow. Nonlinear Differential Equations and Applications NoDEA, pages $1-15,2012$.

[15] F. Dubois and P. LeFloch. Boundary conditions for nonlinear hyperbolic systems of conservation laws. J. Differential Equations, 71(1):93-122, 1988.

[16] R. Eymard, T. Gallouët, and R. Herbin. Finite volume methods. In Handbook of numerical analysis, Vol. VII, Handb. Numer. Anal., VII, pages 713-1020. North-Holland, Amsterdam, 2000.

[17] P. Goatin and S. Scialanga. Well-posedness and finite volume approximations of the LWR traffic flow model with non-local velocity. Netw. Heterog. Media, 11(1):107-121, 2016.

[18] S. Göttlich, S. Hoher, P. Schindler, V. Schleper, and A. Verl. Modeling, simulation and validation of material flow on conveyor belts. Applied Mathematical Modelling, 38(13):3295 - 3313, 2014.

[19] M. Gröschel, A. Keimer, G. Leugering, and Z. Wang. Regularity theory and adjoint-based optimality conditions for a nonlinear transport equation with nonlocal velocity. SIAM J. Control Optim., 52(4):2141-2163, 2014.

[20] S. N. Kružhkov. First order quasilinear equations with several independent variables. Mat. Sb. (N.S.), 81 (123):228-255, 1970.

[21] A. Kurganov and A. Polizzi. Non-oscillatory central schemes for a traffic flow model with arrehenius look-ahead dynamics. Netw. Heterog. Media, 4(3):431-451, 2009.

[22] J. Málek, J. Nečas, M. Rokyta, and M. Růžička. Weak and measure-valued solutions to evolutionary PDEs, volume 13 of Applied Mathematics and Mathematical Computation. Chapman \& Hall, London, 1996.

[23] F. Otto. Initial-boundary value problem for a scalar conservation law. C. R. Acad. Sci. Paris Sér. I Math., 322(8):729-734, 1996.

[24] B. Perthame. Transport equations in biology. Frontiers in Mathematics. Birkhäuser Verlag, Basel, 2007.

[25] D. Serre. Systems of conservation laws. 1 \& 2. Cambridge University Press, Cambridge, 1999. Translated from the 1996 French original by I. N. Sneddon.

[26] A. Sopasakis and M. A. Katsoulakis. Stochastic modeling and simulation of traffic flow: asymmetric single exclusion process with Arrhenius look-ahead dynamics. SIAM J. Appl. Math., 66(3):921-944 (electronic), 2006.

[27] J. Vovelle. Convergence of finite volume monotone schemes for scalar conservation laws on bounded domains. Numer. Math., 90(3):563-596, 2002. 